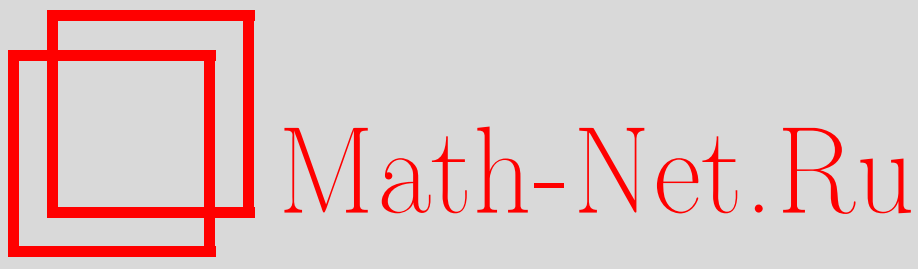

И. А. Ибрагимов, Р. З. Хасьминский, Задачи оценивания коэффициентов стохастических дифференциальных уравнений в частных производных. II, Теория вероятн. и ее примен., 1999, том 44, выпуск 3, 526-554

DOI: https://doi.org/10.4213/tvp802

Использование Общероссийского математического портала Math-Net.Ru подразумевает, что вы прочитали и согласны с пользовательским соглашением http://www . mathnet.ru/rus/agreement

Параметры загрузки:

IP : 3.80 .181 .102

26 апреля 2023 г., 12:58:37

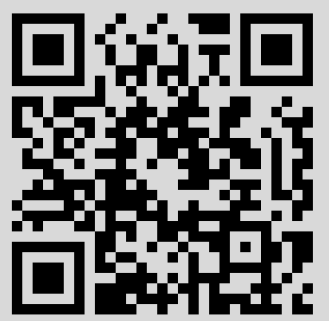


(C) 1999 г. ИБРАГИМОВ И. А. ${ }^{*}$, ХАСЬМИНСКИЙ Р. 3.**

\section{ЗАДАЧИ ОЦЕНИВАНИЯ КОЭФФИЦИЕНТОВ СТОХАСТИЧЕСКИХ ДИФФЕРЕНЦИАЛЬНЫХ УРАВНЕНИЙ В ЧАСТНЫХ ПРОИЗВОДНЫХ. ІІ ${ }^{1)}$}

Как и в первой части (см. [25]), рассматривается задача оценивания функциональных параметров $a_{k}(t, x), \theta(t, x)$ по наблюдению решения $u_{\varepsilon}(t, x)$ стохастического уравнения в частных производных

$$
d u_{\varepsilon}(t)=\sum_{|k| \leqslant 2 p} a_{k} D_{x}^{k} u_{\varepsilon}+\theta d t+\varepsilon d w(t)
$$

$w(t)$ - винеровский процесс. Исследуются вопросы существования состоятельных оценок для $\theta$ и их скорость сходимости к $\theta$ в зависимости от свойств функционального класса $\Theta$, которому априори принадлежит $\theta$.

Ключевые слова и фразы: обратные задачи, стохастические дифференциальные уравнения, статистическое оценивание, непараметрические задачи оценивания.

\section{II. Оценивание свободного члена. Состоятельные оценки. Скорость сходимости}

Здесь мы продолжаем исследование задачи оценивания значения $\Phi(\theta)$ известной функции $\Phi$ в точке $\theta$, которая определяется по наблюдению решения $u_{\varepsilon}(t, x)$ уравнения

$$
d u_{\varepsilon}(t)=L u_{\varepsilon}(t) d t+\theta d t+\varepsilon d w_{Q}(t)
$$

см. ч. I, [25], отказавшись от предположения, что $\Phi^{\prime}(\theta) Q^{1 / 2}$ есть оператор Гильберта-ІІмидта. Конструкции оценок $\S 3$ ч. I в этом случае

${ }^{*}$ С.-Петербургское отделение Математического института им. В. А. Стеклова РАН, ул. Фонтанка, 27, 191011 С.-Петербург, Россия.

** Wayne State University, Detroit, USA.

1) Работа выполнена при частичной поддержке грантов ONR № 00014-95-1-0793, NSF DMS 9600245; первый автор получил также поддержку РФФИ, гранты 96-0100684, 96-15-96199, и INTAS, грант 95-0099. 
теряют смысл, цаже если $\Phi(\theta)$ - линейный ограниченный оператор, и мы интересуемся здесь состоятельным оцениванием $\theta$. Напомним, что нам заранее известно, что $\theta \in \Theta$, где $\Theta$ есть заданное множество функций.

Мы сохраняем введенные в ч. I обозначения для норм в пространствах $L_{p}(G)$ и $\mathscr{L}_{p}=L_{p}([0, T] \times G)$. Как и в ч. I, через $c, C$ обозначаются константы, причем не обязательно одни и те же.

\section{$\S 4$. Состоятельное оценивание и $\varepsilon$-энтропия множества $\Theta$}

4.1. В $\S 2$ ч. I мы произвели формальное сведение нашей задачи $\mathrm{k}$ задаче оценивания сигнала в гауссовском аддитивном шуме. Там же мы описали запас статистик, указав, что все они символически записываются в виде

$$
X(\varphi)=\int_{0}^{T}\left\langle\varphi, d u_{\varepsilon}(t)-L u_{\varepsilon}(t) d t\right\rangle, \quad \varphi \in L_{2}(G)
$$

Благодаря такому сведению, доказательства некоторых из приводимых ниже теорем по существу копируют доказательства соответствующих результатов авторов из [2], [3], посвяпенных оцениванию в гауссовском шуме, и потому в ряде случаев мы опускаем доказательства, ограничиваясь ссылкой на соответствующий результат из [2] или [3].

Мы увидим ниже, что существование состоятельных оценок и их скорость сходимости к $\Phi(\theta)$ зависят от массивности множества $\Phi(\Theta)$, которую в этом параграфе мы характеризуем $\varepsilon$-энтропией, точнее, є-емкостью множества $\Phi(\Theta)$ (см. [1]).

Пусть $M \subseteq \mathscr{L}_{2}$ и $(M, \rho)$ - метрическое пространство с метрикой $\rho$ такой, что

1. $\rho(f, g) \geqslant k\|f-g\|, k>0$;

2. $\rho(f, g)=0 \leftrightarrow\|f-g\|=0$.

Пусть $\Phi: \Theta \rightarrow M$. Назовем оценку $\Phi_{\varepsilon} M$-состоятельной для $\Phi(\theta)$ в $\Theta$, если

$$
\sup _{\theta \in \Theta} \mathbf{P}_{\theta}^{(\varepsilon)}\left\{\rho\left(\Phi_{\varepsilon}, \Phi(\theta)\right)>\delta\right\} \underset{\varepsilon \rightarrow 0}{\longrightarrow} 0
$$

для любого $\delta>0$ (ср. 33, ч. I).

Теорема 4.1. Пусть множество $Q^{-1 / 2} \Theta$ ограничено в $\mathscr{L}_{2}$. $M$-состоятельные в $\Theta$ оченки для $\Phi(\theta)$ могут существовать, лишь если множество $\Phi(\Theta)$ компактно в $M$.

Д о к а з а т е л с т в о теоремы подобно доказательству теоремы 2.1 из [3]. Напомним сперва определение $\delta$-емкости по Колмогорову множества $\Theta$ в $M$. Точки $x_{1}, \ldots, x_{n}$ из $\Theta$ называются $\delta$-различимыми $($ в $(M, \rho))$, если все расстояния $\rho\left(x_{i}, x_{j}\right)>\delta$. Положим $N_{\delta}(\Theta)=\max \left\{n:\left(x_{1}, \ldots, x_{n}\right)-\delta\right.$-различимы $\}$. Логарифм этого числа $C_{\delta}(\Theta,(M, \rho))=C_{\delta}(\Theta)=\ln N_{\delta}(\Theta)$ называется $\delta$-емкостью $\Theta$ (см. [1]). 
Свяжем с множеством' $\Theta$ епе одну величину, определение которой заимствовано из теории информации. Предположим, что в уравнении

$$
d u_{\varepsilon}(t)=L u_{\varepsilon}(t) d t+\theta d t+\varepsilon d w_{Q}(t)
$$

$\theta$ есть случайная функция $(t, x)$, независимая с $w_{Q}$. В этом случае можно определить количество информации по Шеннону, которое содержится в $u_{\varepsilon}$ относительно $\theta$. Напомним, что если $\xi, \eta-$ два случайных элемента с распределениями $P_{\xi}, P_{\eta}$ и совместным распределением $P_{\xi \eta}$, то количество информации в $\xi$ относительно $\eta$

$$
I(\xi, \eta)=\mathbf{E}\left\{\ln \frac{d P_{\xi \eta}}{d P_{\xi} \times d P_{\eta}}(\xi, \eta)\right\}
$$

(см., например, [4]). Определим теперь пропускную способность «канала» (4.1) при ограничении $\Theta$ равенством (относительно определения пропускной способности в теории информации см., например, [5])

$$
\mathscr{C}_{\varepsilon}(\Theta)=\sup I\left(u_{\varepsilon}, \theta\right)
$$

где верхняя грань берется по всем таким распределениям $\theta$, для которых с вероятностью $1 \theta \in \Theta$. Ниже мы рассматриваем $\mathscr{C}_{\varepsilon}(\Theta)$ как характеристику различных возможных множеств $\Theta$ при фиксированном «канале» (4.1).

Лемма 4.1. Пропускная способность $\mathscr{C}_{\varepsilon}(\Theta)$ удовлетворяет неравенству

$$
\mathscr{C}_{\varepsilon}(\Theta) \leqslant \frac{1}{2 \varepsilon^{2}} \sup _{\theta \in \Theta}\left\|Q^{-1 / 2} \theta\right\|^{2} .
$$

Д о к а $з$ а т е ль с т в о использует формулу (3.2), ч. I, для отношения правдоподобия и в дальнейшем развивается как в [2, с. 431].

Теорема 4.2. Имеет место неравенство

$$
\inf _{\widehat{\Phi}} \sup _{\theta} \mathbf{P}_{\theta}\{\rho(\widehat{\Phi}, \Phi(\theta))>\delta\} \geqslant 1-\frac{\mathscr{C}_{\varepsilon}(\Theta)+1}{C_{2 \delta}(\Phi(\Theta))-1} .
$$

Здесь нижняя грань берется по всем возможным оценкам $\widehat{\Phi} \partial л я \Phi(\theta)$.

Д о к а з а т е л ь с т в о см. в [3, теорема 2.2]. Ср. также [2, с. 469].

Теорема 4.1 есть немедленное следствие неравенств (4.3) и (4.4). Действительно, если $\Phi(\Theta)$ не есть компакт, то $C_{2 \delta}(\Phi(\Theta))=\infty$ для всех $\delta>0$, и в силу (4.4) состоятельная оценка не существует.

3 а м е ч а н и е 4.1. Ограниченность множества $Q^{-1 / 2} \Theta$ есть существенное требование. Действительно, параметр $\theta \in \mathbf{R}^{1}$ оценивается состоятельно по наблюдениям статистикой $u_{\varepsilon}$. Поэтому если параметрическое множество $\Theta$ состоит из всех функций $\theta(x) \equiv \theta$, то оценка $\left\langle u_{\varepsilon}(T), \mathbb{I}_{G}\right\rangle T^{-1}(\operatorname{mes} G)^{-1}$ есть несмешенная оценка $\theta$, имеющая дисперсию $\varepsilon^{2} T^{-1}(\operatorname{mes} G)^{-1}$ и, следовательно, состоятельная в $-\infty<\theta<\infty$. 
4.2. Здесь мы приведем несколько теорем, в определенном смысле обратных результатам предыдущего пункта.

Теорема 4.3. Пусть $B$ - нормированное пространство, $а$ $\Phi: \mathscr{L}_{2} \rightarrow B-$ это линейный вполне непрерывный оператор. Если множество $\Theta$ ограничено в $\mathscr{L}_{2}$, то существуют оценки $\Phi_{\varepsilon}$ такие, что для всех $\delta>0$

$$
\sup _{\theta} \mathbf{P}_{\theta}\left\{\left\|\Phi_{\varepsilon}-\Phi(\theta)\right\|_{B}>\delta\right\} \longrightarrow 0 .
$$

Д ок а з а те л ь с т в о см. в [3, теорема 2.3].

Теорема 4.4. Пусть выполнены условия теоремы 4.3, и пусть $B$ - гильбертово пространство. Обозначим $s_{1} \geqslant s_{2} \geqslant \cdots-$ s-числа onepamopa $\Phi$. Tozda

$$
\inf _{\Phi_{\varepsilon}} \sup _{\theta} \mathbf{E}_{\theta}\left\{\left\|\Phi_{\varepsilon}-\Phi(\theta)\right\|\right\} \leqslant \sup _{\theta} \inf _{n}\left(s_{n+1}\|\theta\|+\varepsilon\left\|Q^{1 / 2}\right\| \sum_{1}^{n} s_{j}^{2}\right)^{1 / 2} .
$$

Д о к а з а т е л ь с т в о см. в [3], замечание к теореме 2.3.

Теорема 4.5. Пусть иножество $Q^{-1 / 2} \Theta$ компактно в $\mathscr{L}_{2} ;$ пусть функция $\Phi: \Theta \rightarrow M$, где $(M, \rho)$ - метрическое пространство, равномерно непрерывна в $\Theta$. Тогда существуют $M$-состоятельные оченки dлst $\theta$.

Доказ а тель с т в о сходно с доказательством теоремы 2.4 из $[3]$.

Определим модуль непрерывности функции $\Phi$ равенством

$$
\omega(\delta)=\sup \left\{\rho\left(\Phi\left(\theta_{1}\right), \Phi\left(\theta_{2}\right)\right): \theta_{1}, \theta_{2} \in \Theta,\left\|\theta_{1}-\theta_{2}\right\| \leqslant \delta\right\} .
$$

В множестве $Q^{-1 / 2} \Theta$ выберем сеть из $n=\exp \left\{C_{\delta}\left(Q^{-1 / 2} \Theta\right)\right\} \delta$-различимых точек $t_{1}=Q^{-1 / 2} \theta_{1}, \ldots, t_{n}=Q^{-1 / 2} \theta_{n}$. Точки $\left\{t_{\nu}\right\}$ образуют $\delta$-сеть в $Q^{-1 / 2} \Theta$. Поэтому, если $\theta-$ произвольная точка из $\Theta$ и $t=Q^{-1 / 2} \theta$, найдется точка $t_{j}=Q^{-1 / 2} \theta_{j}$ такая, что $\left\|t-t_{j}\right\| \leqslant \delta$. Но тогда и

$$
\left\|\theta-\theta_{j}\right\| \leqslant\left\|Q^{1 / 2}\right\|\left\|Q^{-1 / 2} \theta-Q^{-1 / 2} \theta_{j}\right\| \leqslant \delta\left\|Q^{1 / 2}\right\| \text {. }
$$

Рассмотрим теперь статистики

$$
\varphi_{j}\left(u_{\varepsilon}\right)=\frac{1}{\varepsilon^{2}} \int_{0}^{T}\left\langle Q^{-1 / 2} \theta_{j}, Q^{-1 / 2}\left(d u_{\varepsilon}(t)-L u_{\varepsilon}(t) d t\right)\right\rangle-\frac{1}{2 \varepsilon^{2}}\left\|Q^{-1 / 2} \theta_{j}\right\|^{2},
$$

представляющие собой $\ln \left(d \mathbf{P}_{\theta_{j}} / d \mathbf{P}_{0}\right)$, и определим ощенку $\Phi_{\varepsilon}^{*}$ следующим образом:

$$
\Phi_{\varepsilon}^{*}=\Phi\left(\theta_{i}\right), \quad \text { если } \max _{j} \varphi_{j}=\varphi_{i}
$$

(если максимум достигается в нескольких точках, мы выбираем одну из них, безразлично какую). 
Tогда

$$
\begin{gathered}
\sup _{\theta} \mathbf{P}_{\theta}\left\{\rho\left(\Phi_{\varepsilon}^{*}, \Phi(\theta)\right)>\omega\left(3\|Q\|^{1 / 2} \delta\right)+\omega\left(\|Q\|^{1 / 2} \delta\right)\right\} \\
\leqslant \sqrt{\frac{\varepsilon}{\delta}} \exp \left\{\frac{1}{2} C_{\delta}\left(Q^{-1 / 2} \Theta\right)-\frac{\delta^{2}}{16 \varepsilon^{2}}\right\} .
\end{gathered}
$$

Утверждение теоремы немедленно следует из (4.5). Само же неравенство (4.5) доказывается так же, как аналогичное ему неравенство (2.4) из [3], и мы опустим доказательство.

3 а м е ч а н и 4.2. Допустим, что оцениванию подлежит функция $\Phi=\theta$. Естественной мерой точности оценки $\Phi_{\varepsilon}$ служит величина $\left\|Q^{-1 / 2}\left(\Phi_{\varepsilon}-\theta\right)\right\|$. Из теорем 4.1 и 4.5 вытекает следуюпий результат.

Теорема 4.6. Пусть миожество $Q^{-1 / 2} \Theta$ ограничено в $\mathscr{L}_{2}$. Тогда

$$
\inf _{\Phi_{\varepsilon}} \sup _{\theta} \mathbf{P}_{\theta}\left\{\left\|Q^{-1 / 2}\left(\Phi_{\varepsilon}-\theta\right)\right\|>\delta\right\} \longrightarrow 0
$$

для всех $\delta>0$ в том и только том случае, если множество $Q^{-1 / 2} \Theta$ компактно в $\mathscr{L}_{2}$.

Действительно, эту задачу можно трактовать как задачу оценивания $\Phi(\theta)=Q^{-1 / 2} \theta$ в $\mathscr{L}_{2}$-метрике. По теореме 4.1 множество $\Phi(\Theta)$ компактно. В силу же теоремы 4.5 состоятельное оценивание возможно, так как если $Q^{-1 / 2} \Theta$ - компакт, то функция $\Phi(\theta)$ равномерно непрерывна на $\Theta$ в метрике $\mathscr{L}_{2}$.

4.3. Неравенства (4.4) и (4.5), хотя и выглядят очень грубыми, в ряде случаев позволяют получить асимптотически точные результаты в отношении порядка сходимости оценок к $\Phi(\theta)$. В качестве примера рассмотрим задачу оценивания $\theta$ в $\mathscr{L}_{2}$-норме в предположении, что оператор $Q^{-1 / 2}$ ограничен. Для простоты мы всюду ниже, не оговаривая этого особо, будем считать $Q=I$.

Пусть $V$ - гильбертово пространство, $\left\{v_{j}\right\}$ - ортогональные элементы в нем и $a_{j} \uparrow$ - монотонная последовательность положительных чисел. Подмножества $V$ вида

$$
\Sigma=\Sigma\left\{\left\{a_{n}\right\}\right\}=\left\{v: v \in V, v=\sum_{1}^{\infty} x_{j} v_{j}, \sum_{1}^{\infty} x_{j}^{2} a_{j}^{2} \leqslant 1\right\}
$$

будем называть эллипсоидами $\left(a_{j}^{-1}-\right.$ длины полуосей). Заметим, что $\left\{v_{j}\right\}$ не обязательно базис в $V$, так что $\Sigma$ может быть и эллипсоидальным циском.

В определении эллипсоида допускаются значения $a_{n}=\infty$, причем $x_{j}^{2} \cdot \infty=\infty, x_{j}>0,0 \cdot \infty=0$. 
Теорема 4.7. Пусть $\Theta=\Theta\left\{\left\{a_{n}\right\}\right\}-$ эллuncoud в $\mathscr{L}_{2}$. Тогдa

1) если $a_{n} \asymp n^{\beta}(\ln n)^{\gamma}, \beta>0,-\infty<\gamma<\infty$, mo

$$
\Delta_{\varepsilon}(\Theta)=\inf _{\theta_{\varepsilon}} \sup _{\theta} \mathbf{E}_{\theta}\left\|\theta_{\varepsilon}-\theta\right\|^{p} \asymp \varepsilon^{2 \beta p /(2 \beta+1)}\left(\ln \frac{1}{\varepsilon}\right)^{-\gamma p /(2 \beta+1)},
$$

2) если начиная с некоторого места $a_{n}=\infty$, mо

$$
\Delta_{\varepsilon}(\Theta) \asymp \varepsilon^{p} .
$$

Д о к а 3 а т е л ь с т в о. Докажем сначала оценку снизу.

Лемма 4.2. В условиях теоремы 4.1 имеет место неравенстьо

$$
\mathbf{E}_{\theta} l\left(\rho\left(\Phi_{\varepsilon}, \Phi(\theta)\right)\right) \geqslant \sup _{\delta>0} l(\delta)\left(1-\frac{\mathscr{C}_{\varepsilon}(\Theta)+1}{C_{2 \delta}(\Theta)-1}\right),
$$

какова бы ни была неотрицательная монотонно неубывающая функция $l(y)$, определенная для $y>0$.

Действительно, левая часть (4.6) не меньше

$$
\int_{\{\rho>\delta\}} l\left(\rho\left(\Phi_{\varepsilon}, \Phi(\theta)\right)\right) d \mathbf{P}_{\theta} \geqslant l(\delta) \mathbf{P}_{\theta}\left\{\rho\left(\Phi_{\varepsilon}, \Phi(\theta)\right)>\delta\right\},
$$

и нам достаточно сослаться на (4.4).

Чтобы оценить емкости в (4.6), рассмотрим сперва $n$-мерный диск радиуса $r$ в $\mathscr{L}_{2}$ :

$$
B_{r}=\left\{h \in \mathscr{L}_{2}: h=\sum_{1}^{n} h_{j} v_{j}, \sum_{1}^{n} h_{j}^{2} \leqslant r^{2}\right\},
$$

и оценим $C_{\delta}\left(B_{r}\right)$. Очевидно, $C_{\delta}\left(B_{r}\right)$ совпадает с $\delta$-емкостью $n$-мерного евклидова шара радиуса $r$, который мы тоже обозначаем $B_{r}$. Если $x_{1}, \ldots, x_{m}-\delta$-различимые точки из $B_{r}$, то шары радиуса $\delta$ с центрами в $x_{j}$ покрывают $B_{r}$, и, значит, их суммарный объем больше объема $B_{r}$. Следовательно, $m \delta^{n} \geqslant r^{n}$ и $C_{\delta}\left(B_{r}\right) \geqslant n \ln (r / \delta)$. По лемме 4.1

$$
\mathscr{C}_{\varepsilon}\left(B_{r}\right) \leqslant \frac{r^{2}}{2 \varepsilon^{2}}
$$

Циск размерности $n$ и радиуса $r=a_{n}^{-1}$ :

$$
B_{r}=\left\{\theta: \theta=\sum_{1}^{n} c_{j} v_{j}, \sum_{1}^{n} c_{j}^{2} \leqslant a_{n}^{-2}\right\} \subseteq \Theta .
$$

Поэтому, какова бы ни была оценка $\theta_{\varepsilon}$,

$$
\begin{aligned}
\sup _{\theta \in \Theta} \mathbf{E}_{\theta}\left\|\theta_{\varepsilon}-\theta\right\|^{p} & \geqslant \sup _{\theta \in B_{r}} \mathbf{E}_{\theta}\left\|\theta_{\varepsilon}-\theta\right\|^{p} \geqslant \delta^{p}\left(1-\frac{\mathscr{C}_{\varepsilon}\left(B_{r}\right)+1}{C_{2 \delta}\left(B_{r}\right)-1}\right) \\
& \geqslant \delta^{p}\left(1-\frac{r^{2} / 2 \varepsilon^{2}+1}{n \ln (r / \delta)-1}\right) .
\end{aligned}
$$


Выбирая здесь сперва $\delta=\frac{1}{2} a_{n}^{-1}$, а затем определяя $N$ из соотноџения

$$
N=\min \left\{n: \frac{2^{-1} a_{n}^{-2} \varepsilon^{-2}+1}{n \ln 2-1} \leqslant \frac{1}{2}\right\},
$$

получим $\Delta_{\varepsilon}(\Theta) \geqslant 4 a_{N}^{-p}$. В частьности, в условиях п. 1) теоремы $N \asymp$ $\varepsilon^{-2 /(2 \beta+1)}(\ln (1 / \varepsilon))^{-2 \gamma /(2 \beta+1)}$, и потому

$$
\Delta_{\varepsilon}(\Theta) \geqslant c \varepsilon^{2 \beta p /(2 \beta+1)}\left(\ln \frac{1}{\varepsilon}\right)^{-\gamma p /(2 \beta+1)},
$$

где $c>0$.

Оценка сверху. Известные результаты о поведении $\varepsilon$-энтропии эллипсоидов (см. [1]) дают, что в условиях п. 1) теоремы

$$
C_{\delta}(\Theta) \asymp\left(\frac{1}{\delta}\right)^{1 / \beta}\left(\ln \frac{1}{\delta}\right)^{-\gamma / \beta} .
$$

В силу неравенства (4.5) найдутся оценки $\theta_{\varepsilon}$ такие, что для любого $\delta>0$

$$
\begin{aligned}
\mathbf{E}_{\theta}\left\|\theta_{\varepsilon}-\theta\right\|^{p} \leqslant & (4 \delta)^{p}+\int_{4 \delta}^{\infty} y^{p} d \mathbf{P}_{\theta}\left\{\left\|\theta_{\varepsilon}-\theta\right\|>y\right\} \\
\leqslant & (4 \delta)^{p}+(4 \delta)^{p-1 / 2} \sqrt{\varepsilon} \exp \left\{\frac{1}{2} C_{2 \delta}(\Theta)-\frac{\delta^{2}}{16 \varepsilon^{2}}\right\} \\
& +p \sqrt{\frac{\varepsilon}{\delta}} \int_{4 \delta}^{\infty} y^{p-3 / 2} \exp \left\{\frac{1}{2} C_{2 y}(\Theta)-\frac{y^{2}}{4^{2} \varepsilon^{2}}\right\} d y
\end{aligned}
$$

Выбирая здесь $\delta \asymp \varepsilon^{2 \beta /(2 \beta+1)}(\ln (1 / \varepsilon))^{-\gamma /(2 \beta+1)}$, найдем, что

$$
\Delta_{\varepsilon}(\Theta) \leqslant c \varepsilon^{2 \beta p /(2 \beta+1)}\left(\ln \frac{1}{\varepsilon}\right)^{-\gamma p /(2 \beta+1)},
$$

где $c$ - некоторая постоянная.

Первая часть теоремы доказана. Вторая часть ее очевидна. Более того, в условиях, когда $a_{k}=\infty, k>n$, оператор $\Phi^{\prime}(\theta) P_{H}$ конечномерен, и возможно говорить об асимптотически эффективном оценив|ании. Асимптотически эффективной будет оценка

$$
\theta_{\varepsilon}=\sum_{1}^{n} \int_{0}^{T}\left\langle v_{j}, d u_{\varepsilon}(t)-L u_{\varepsilon}(t) d t\right\rangle \cdot v_{j}
$$

П р и м е р 4.1. Пространства гладких функций. В этом примере мы полагаем, что $G$ есть куб $[0,1]^{d}$ и $\Theta$ состоит из функций $\theta(x)$, зависящих только от $x$, периодических с периодом 1 по каждому аргументу и обладающих определенной гладкостью в $L_{2}$. Мы определим классы гладких функций, следуя работе Бабенко [6] (см. также [7], [8]). 
Пусть $P(z)-$ полином от $d$ переменных $z=\left(z_{1}, \ldots, z_{d}\right)$. Положим

$$
D=\left(\frac{1}{i} \frac{\partial}{\partial x_{1}}, \ldots, \frac{1}{i} \frac{\partial}{\partial x_{d}}\right)
$$

Определим класс $W_{2}^{P}$ периодических функций как множество таких функций $\theta$, что $|P(D) \theta|_{2} \leqslant 1$. Разлагая функцию $\theta(x)$ в ряд Фурье

$$
\theta(x)=\sum_{j} \theta_{j} \exp \{2 \pi i(x, j)\},
$$

найдем, что $W_{2}^{P}$ состоит из функций $\theta$ таких, что

$$
\sum_{j}|P(2 \pi j)|^{2}\left|\theta_{j}\right|^{2} \leqslant 1
$$

и представляет собой эллипсоид в $L_{2}$. Поскольку $P(z)$ может обращаться в нуль, эллипсоид может оказаться некомпактным; поэтому, определяя $\Theta$ через $W_{2}^{P}$, мы должны наложить дополнительные ограничения на те $\theta_{j}$, для которых $P(2 \pi j)=0$. В частности, в этом пункте мы полагаем

$$
\Theta=W_{2}^{P} \cap\left\{\theta: \theta_{j}=0, \text { если } P(2 \pi j)=0\right\} .
$$

Конечно, ничего не изменится, если $\theta_{j} \neq 0$ для конечного множества $j$ таких, что $P(2 \pi j)=0$.

Множество $\Theta$ есть эллипсоид, длины полуосей которого определяются требованием, что область $\left\{x: c<|P(2 \pi x)| \leqslant a_{n}\right\}, c=c(P)>0$, должна содержать $n$ точек $\left(j_{1}, \ldots, j_{d}\right)$ с целыми координатами. Поэтому во многих случаях асимптотическое поведение $a_{n}$ вычисляется с помощью соотношений

$$
n \sim \operatorname{Vol}\left\{x ; c<|P(2 \pi x)| \leqslant a_{n}\right\} \sim \int_{c<|P(2 \pi x)| \leqslant a_{n}} d x
$$

(функция $a_{n}$ определяется из соотношений, что область $\{x: c<$ $\left.|P(2 \pi x)| \leqslant a_{n}\right\}$ должна содержать $n$ целых точек $\left.j\right)$.

Рассмотрим два специальных примера.

1. Пусть полином

$$
P(z)=\left(\sum_{1}^{d} c_{j} z_{j}^{\beta_{j}}\right)^{k},
$$

где все $c_{j}>0, \beta_{j}$ - целые четные, $k$ - целое.

Если $\left\{\theta_{j}\right\}$ - коэффициенты Фурье функции $\theta$, то множество $\Theta$ определяется как множество функций $\theta$, для которых

$$
\left.\left.\sum_{j=\left(j_{1}, \ldots, j_{d}\right)}\left|\sum_{r=1}^{d} c_{j}\right| 2 \pi j_{r}\right|^{\beta_{j}}\right|^{2 k}\left|\theta_{j}\right|^{2} \leqslant 1 .
$$

При такой записи множество $\Theta$ корректно определено уже при произвольных $\beta_{j}>0, k>0$, и мы рассматриваем все такие множества $\Theta$. 
(В частности, при $k=\frac{1}{2}$ условие (4.8) означает, что $\theta(x)$ имеет в $L_{2}$ обобщенные производные по $x_{j}$ порядка $\beta_{j} / 2$.) Из формулы (4.7) следует, что

$$
n \sim c(a(n))^{k^{-1} \sum_{1}^{d} \beta_{r}^{-1}}, \quad a(n) \sim C n^{k \beta}, \quad \frac{1}{\beta}=\frac{1}{\beta_{1}}+\cdots+\frac{1}{\beta_{d}} .
$$

В силу теоремы 4.7, если $\Theta$ определено равенством (4.8), то

$$
\Delta_{\varepsilon}(\Theta)=\inf _{\theta_{\varepsilon}} \sup _{\theta} \mathbf{E}_{\theta}\left|\theta_{\varepsilon}-\theta\right|_{2}^{p} \asymp \varepsilon^{2 p k \beta /(2 k \beta+1)} .
$$

2. Пусть теперь полином $P(z)=z_{1}^{\beta_{1}} \cdots z_{d}^{\beta_{d}}$. Множество $\Theta$ определяется неравенством

$$
\sum\left|\theta_{j}\right|^{2}\left|2 \pi j_{1}\right|^{2 \beta_{1}} \cdots\left|2 \pi j_{d}\right|^{2 \beta_{d}} \leqslant 1
$$

и дополнительными условиями: $\theta_{j}=0$, если какая-нибудь из компонент вектора $j$ есть ноль. Как и выше, можно считать, что в определении (4.9) $\beta_{j}$ - произвольные положительные числа, и (4.9) означает ограниченность в $L_{2}$ обобщенных смешанных производных порядка $\left(\beta_{1}, \ldots, \beta_{d}\right)$. Чтобы выписать асимптотические формулы для полуосей эллипсоида $\Theta$, расположим числа $\beta_{j}$ в порядке возрастания. Пусть для определенности $\beta=\beta_{1}=\cdots=\beta_{l}<\beta_{l+1} \leqslant \cdots \leqslant \beta_{d}$. Из (4.7) следует, что $n \sim c(a(n))^{1 / \beta}(\ln a(n))^{l-1}, \beta=\min \beta_{j}$. Поэтому $a(n) \sim C n^{\beta}(\ln n)^{-(l-1) \beta}$, и в силу теоремы 4.7

$$
\Delta_{\varepsilon}(\Theta) \asymp \varepsilon^{2 p \beta /(2 \beta+1)}\left(\ln \frac{1}{\varepsilon}\right)^{p \beta(l-1) /(2 \beta+1)} .
$$

\section{§5. Состоятельные оценки и поперечники}

5.1. Пусть $B-$ банахово пространство с нормой $\|\cdot\|$. Нам понадобится определение двух следующих поперечников множеств $\Sigma \subseteq B$ (подробнее об этих и других поперечниках см. [9], [10]).

1. П о п е р е ч н и к и по К ол м ог ор ов у $d_{n}(\Sigma), n=0,1, \ldots$, множества $\Sigma$ в $B$ определяются равенствами

$$
d_{n}(\Sigma)=\inf _{M_{n}} \sup _{x \in \Sigma} \inf _{y \in M_{n}}\|x-y\|,
$$

где $M_{n}$ пробегают все $n$-мерные линейные подмногообразия $B$.

2. II о п е р е ч н и к и по Б е р нш те й н у $b_{n}(\Sigma), n=0,1, \ldots$, множества $\Sigma$ в $B$ определяются равенствами

$$
b_{n}(\Sigma)=\sup _{M_{n+1}} \sup _{r>0}\left\{r: r O \cap M_{n+1} \subseteq \Sigma\right\}
$$

где $O$ обозначает единичный шар в $B$, а $M_{n}$ пробегает все линейные $n$-мерные подмногообразия в $B$. 
Ниже мы для простоты, не оговаривая это особо, рассматриваем поперечники лишь в гильбертовых пространствах $L_{2}$ и $\mathscr{L}_{2}$.

Теорема 5.1. Существуют такие постоянные $c_{p}$, что

$$
\Delta_{\varepsilon}(\Theta)=\inf _{\theta_{\varepsilon}} \sup _{\theta} \mathbf{E}_{\theta}\left\|\theta_{\varepsilon}-\theta\right\|^{p} \leqslant c_{p} \inf _{n}\left((\varepsilon \sqrt{n})^{p}+\left(d_{n}(\Theta)\right)^{p}\right),
$$

где $d_{n}(\Theta)$ - n-й поперечник Колмогорова множества $\Theta$ в $\mathscr{L}_{2}$.

Д о к а з а т ел в с т в о. Фиксируем целое число $n$ и подберем линейное многообразие $M_{n}$ размерности $n$ так, чтобы расстояние $\Theta$ от $M_{n}$ не превышало $d_{n}(\Theta)+\gamma, \gamma \geqslant 0$. Без потери общности можно считать, что $M_{n}$ есть подпространство в $\mathscr{L}_{2}$. Пусть $\varphi_{1}, \ldots, \varphi_{n}$ - ортонормальный базис в $M_{n}$. Определим оценки

$$
\begin{aligned}
\theta_{n} & =\sum_{1}^{n} \varphi_{j} \cdot \int_{0}^{T}\left\langle\varphi_{j}, d u_{\varepsilon}(t)-L u_{\varepsilon}(t) d t\right\rangle \\
& =\sum_{1}^{n} \varphi_{j}\left(\varphi_{j}, \theta\right)+\varepsilon \sum_{1}^{n} \varphi_{j} \int_{0}^{T}\left\langle\varphi_{j}, d w_{Q}(t)\right\rangle .
\end{aligned}
$$

Первое слагаемое справа есть проекция $\theta$ на $M_{n}$ и

$$
\left\|\theta-\sum_{1}^{n} \varphi_{j}\left(\varphi_{j}, \theta\right)\right\| \leqslant d_{n}(\Theta)+\gamma .
$$

Далее, полагая $\xi_{j}=\int_{0}^{T}\left\langle\varphi_{j}, d w_{Q}(t)\right\rangle$, найдем, что

$$
\left\|\sum_{1}^{n} \xi_{j} \varphi_{j}\right\|^{2}=\sum_{1}^{n} \xi_{j}^{2}
$$

и потому для всех $p>0$

$$
\mathbf{E}\left\|\sum_{1}^{n} \xi_{j} \varphi_{j}\right\|^{p} \leqslant c_{p}\left(\sum_{1}^{n} \mathbf{E} \xi_{j}^{2}\right)^{p / 2} \leqslant c_{p}\left(\sum_{1}^{n}\left\|Q^{1 / 2} \varphi_{j}\right\|^{2}\right)^{p / 2}
$$

Следовательно,

$$
\begin{aligned}
\mathbf{E}_{\theta}\left\|\theta_{n}-\theta\right\|^{p} & \leqslant 2^{p-1}\left(\left(d_{n}(\Theta)+\gamma\right)^{p}+\varepsilon^{p} \mathbf{E}\left\|\sum_{1}^{n} \xi_{j} \varphi_{j}\right\|^{p}\right) \\
& \leqslant c_{p}\left(\left(d_{n}(\Theta)+\gamma\right)^{p}+\varepsilon^{p}\left(\sum_{1}^{n}\left\|Q^{1 / 2} \varphi_{j}\right\|^{2}\right)^{p / 2}\right)
\end{aligned}
$$

Последняя сумма справа не больше $n\|Q\|$, и мы приходим к утверждению теоремы.

3 а м е ч н и е 5.1. Границу теоремы 5.1 можно слегка усилить следующим образом. Обозначим $S_{N}$ множество всех операторов Гильберта-Шмидта $S: \mathscr{L}_{2} \rightarrow \mathscr{L}_{2}$ с нормой Гильберта-Пмидта 
$\left|S_{N}\right|_{2} \leqslant \sqrt{N}$. Определим поперечники $h_{N}(\Theta)$ равенством

$$
h_{N}(\Theta)=\inf _{M} \sup _{\theta} \inf _{y \in M}\|\theta-y\|
$$

где $M$ пробегает все подмножества $\mathscr{L}_{2}$, представимые в виде

$$
M=S \mathscr{L}_{2}+f, \quad S \in S_{N}, f \in \mathscr{L}_{2} .
$$

Те же самые рассуждения, что были использованы при доказательстве теоремы 5.1 , дают

$$
\inf _{\theta_{\varepsilon}} \sup _{\theta} \mathbf{E}_{\theta}\left\|\theta_{\varepsilon}-\theta\right\|^{p} \leqslant c_{p} \inf _{n}\left(\left(h_{n}(\Theta)\right)^{p}+(\varepsilon \sqrt{n})^{p}\right) .
$$

Это неравенство сильнее, чем $(5.1)$, так как $h_{n}(\Theta) \leqslant d_{n}(\Theta)$.

Неравенство (5.3) можно еще переписать следуюшим образом. Пусть $\Psi=\left\{\psi_{j}\right\}-$ полная ортонормальная система в $\mathscr{L}_{2}$, пусть $\Lambda=\left\{\lambda_{j}\right\}-$ числовая последовательность с $\sum \lambda_{j}^{2}<\infty$. Рассмотрим всевозможные линейные оценки

$$
l=\sum \lambda_{j} \int_{0}^{T}\left\langle\psi_{j}, d u_{\varepsilon}(t)-L u_{\varepsilon}(t) d t\right\rangle
$$

Мы найдем тогда, что

$$
\begin{aligned}
& \inf _{\theta_{\varepsilon}} \sup _{\theta} \mathbf{E}_{\theta}\left\|\theta_{\varepsilon}-\theta\right\|^{p} \leqslant \inf _{l} \sup _{\theta} \mathbf{E}_{\theta}\|l-\theta\|^{p} \\
& \quad \leqslant c_{p} \inf _{\Lambda, \Psi} \sup _{\theta}\left\{\left(\sum_{j}\left(1-\lambda_{j}\right)^{2}\left(\theta, \psi_{j}\right)^{2}\right)^{p / 2}+\varepsilon^{p}\left(\sum_{j} \lambda_{j}^{2}\right)^{p / 2}\right\} .
\end{aligned}
$$

В частности,

$$
\inf _{\theta_{\varepsilon}} \sup _{\theta} \mathbf{E}_{\theta}\left\|\theta_{\varepsilon}-\theta\right\|^{2} \leqslant c \inf _{\Lambda, \Psi} \sup _{\theta}\left\{\left(1-\lambda_{j}\right)^{2}\left(\theta, \psi_{j}\right)^{2}+\varepsilon^{2} \sum \lambda_{j}^{2}\right\} .
$$

5.2. Теорема 5.2. Пусть оператор $Q^{-1}$ ограничен. Существуют такие положительные постоянные $C_{p}>0$, ито

$$
\inf _{\theta_{\varepsilon}} \sup _{\theta} \mathbf{E}_{\theta}\left\|\theta_{\varepsilon}-\theta\right\|^{p} \geqslant C_{p} \varepsilon^{p}\left(\sup \left\{n: \frac{b_{n}(\Theta)}{\varepsilon \sqrt{n+1}} \geqslant 1\right\}\right)^{p / 2}, \quad p \geqslant 1 .
$$

Д ок а 3 а т е л в с т в о. Ограничимся случаем $Q=I, p \geqslant 2$. Фиксируем целое число $n$ и предположим для определенности, что шар $B_{n} \subseteq \Theta \cap M_{n+1}$ максимального радиуса $b_{n}$ имеет своим центром начало координат. Очевидно,

$$
\sup _{\theta} \mathbf{E}_{\theta}\left\|\theta_{\varepsilon}-\theta\right\|^{p} \geqslant \sup _{\theta \in B_{n}}\left\|\theta_{\varepsilon}-\theta\right\|^{p}
$$


Пусть $\varphi_{1}, \ldots, \varphi_{n+1}$ - ортонормальный базис в $M_{n+1}$. Записывая $\theta \in B_{n}$ в виде $\theta=\sum \theta_{j} \varphi_{j}$, найдем

$$
\frac{d \mathbf{P}_{\theta}}{d \mathbf{P}_{0}}\left(u_{\varepsilon}\right)=\exp \left\{\frac{1}{\varepsilon^{2}} \sum_{1}^{n+1} \theta_{j} \int_{0}^{T}\left\langle\varphi_{j}, d u_{\varepsilon}(t)-L u_{\varepsilon}(t) d t\right\rangle-\frac{1}{2 \varepsilon^{2}} \sum_{1}^{n+1} \theta_{j}^{2}\right\} .
$$

Поэтому в задаче оценивания $\theta \in B_{n}$ статистики

$$
X_{j}=\int_{0}^{T}\left\langle\varphi_{j}, d u_{\varepsilon}(t)-L u_{\varepsilon}(t) d t\right\rangle
$$

образуют семейство достаточных статистик, и нам достаточно рассматривать лишь оценки вида $\hat{\theta}\left(X_{1}, \ldots, X_{n+1}\right)$.

Далее,

$$
X_{j}=\theta_{j}+\varepsilon \int_{0}^{T}\left\langle\varphi_{j}, d w(t)\right\rangle=\theta_{j}+\varepsilon \xi_{j}
$$

где $\xi_{1}, \ldots, \xi_{n+1}$ - независимые одинаково распределенные величины с параметрами $(0,1)$. Куб $\Gamma_{n}=\left\{\theta=\left(\theta_{1}, \ldots, \theta_{n+1}\right):\left|\theta_{j}\right| \leqslant b_{n} / \sqrt{n+1}\right\} \subseteq B_{n}$. Поэтому

$$
\begin{aligned}
\sup _{\theta \in B_{n}} \mathbf{E}_{\theta}\left\|\theta_{\varepsilon}-\theta\right\|^{2} & \geqslant \sup _{\theta \in \Gamma_{n}} \mathbf{E}_{\theta}\left|\theta-\theta_{\varepsilon}\left(X_{1}, \ldots, X_{n+1}\right)\right|^{2} \\
& \geqslant\left(\operatorname{mes} \Gamma_{n}\right)^{-1} \int_{\Gamma_{n}} \mathbf{E}_{\theta}\left|\theta-\theta_{\varepsilon}\left(X_{1}, \ldots, X_{n+1}\right)\right|^{2} d \theta .
\end{aligned}
$$

По теореме Ченцова [16] для всех $\theta_{\varepsilon}$

$$
\begin{gathered}
\left(\operatorname{mes} \Gamma_{n}\right)^{-1} \int_{\Gamma_{n}} \mathbf{E}_{\theta}\left|\theta-\theta_{\varepsilon}\left(X_{1}, \ldots, X_{n+1}\right)\right|^{2} d \theta \\
\geqslant \varepsilon^{2}\left(1-\frac{\varepsilon \sqrt{n+1}}{b_{n}} \operatorname{th}\left(\frac{b_{n}}{\varepsilon \sqrt{n+1}}\right)\right)
\end{gathered}
$$

Функция th $x / x<1$ всюду, кроме точки $x=0, \lim _{x \rightarrow \infty}(\operatorname{th} x / x)=0$, следовательно,

$$
\inf _{\theta_{\varepsilon}} \sup _{\theta} \mathbf{E}_{\theta}\left\|\theta_{\varepsilon}-\theta\right\|^{2} \geqslant \varepsilon^{2} \inf _{x \geqslant 1}\left(1-\frac{\operatorname{th} x}{x}\right) \sup \left\{n: \frac{b_{n}(\theta)}{\varepsilon \sqrt{n+1}} \geqslant 1\right\}
$$

что эквивалентно утверждению теоремы для $p=2$. Если $p>2$, то $\mathbf{E}_{\theta}\left\|\theta_{\varepsilon}-\theta\right\|^{p} \geqslant \mathbf{E}_{\theta}^{p / 2}\left\|\theta_{\varepsilon}-\theta\right\|^{2}$. Случай $p<2$ трактуется сходным образом, но уже требует отдельной оценки интеграла

$$
\frac{1}{\operatorname{mes} \Gamma_{n}} \int_{\Gamma_{n}} \mathbf{E}\left|\theta_{\varepsilon}\left(X_{1}, \ldots, X_{n+1}\right)-\theta\right| d \theta .
$$


5.3. Примеры. Пусть $\Theta$ есть эллипсоид $\Theta\left(a_{1}, \ldots\right)$. Тогда $d_{n}(\Theta)=$ $b_{n}(\Theta)=a_{n+1}^{-1}$. Впрочем, нам будет достаточно и совсем очевидных неравенств $d_{n} \leqslant a_{n+1}^{-1}$ и $b_{n} \leqslant a_{n+1}^{-1}$. Цопустим, что при $n \rightarrow \infty a_{n} \asymp n^{\beta}(\ln n)^{\gamma}$. Тогда из теоремы 5.1 следует, что

$$
\Delta_{\varepsilon}(\Theta) \leqslant c \min _{x}\left(\left(x^{-\beta}(\ln x)^{-\gamma}\right)^{p}+\varepsilon^{p} x^{p / 2}\right)=c \varepsilon^{2 p \beta /(2 \beta+1)}\left(\ln \frac{1}{\varepsilon}\right)^{-p \gamma /(2 \beta+1)},
$$

а из теоремы 5.2 , что

$$
\begin{aligned}
\Delta_{\varepsilon}(\Theta) & \leqslant c\left(\varepsilon^{2} \sup _{x}\left\{x: x^{\beta+1 / 2} \ln ^{\gamma} x \leqslant \frac{1}{\varepsilon}\right\}\right)^{p / 2} \\
& =c \varepsilon^{2 p \beta /(2 \beta+1)}\left(\ln \frac{1}{\varepsilon}\right)^{-p \gamma /(2 \beta+1)}, \quad c>0 .
\end{aligned}
$$

Таким образом, мы снова пришли к теореме 4.7: если $a_{n} \asymp n^{\beta}(\ln n)^{\gamma}$, то

$$
\Delta_{\varepsilon}(\Theta) \asymp \varepsilon^{2 p \beta /(2 \beta+1)}\left(\ln \frac{1}{\varepsilon}\right)^{-p \gamma /(2 \beta+1)} .
$$

Теорема 5.3. Пусть оператор $Q^{-1}$ ограничен. Пусть $\Theta=$ $\Theta\left(\left\{a_{n}\right\}\right)$ ecmь эллипсоид $c \ln a_{n} \asymp n^{\gamma}$. Тогдa

$$
\Delta_{\varepsilon}(\Theta) \asymp \varepsilon^{p} \cdot \ln ^{p /(2 \gamma)} \frac{1}{\varepsilon} .
$$

Д о к а 3 а т е л ь с т в о. В условиях теоремы $d_{n}(\Theta) \leqslant 2^{-c_{1} n^{\gamma}}, c_{1}>$ 0 . Полагая в $(5.1) n \asymp\left(\ln \varepsilon^{-1}\right)^{1 / 2}$, найдем, что $\Delta_{\varepsilon}(\Theta) \leqslant c \varepsilon^{p}\left(\ln \varepsilon^{-1}\right)^{p /(2 \gamma)}$. Далее, в условиях теоремы $b_{n}(\Theta) \geqslant 2^{-c_{2} n^{\gamma}}$, и, выбирая в (5.6) $n \sim$ $\left(\ln \varepsilon^{-1}\right)^{1 / \gamma}$, мы придем к утверждению теоремы.

П р и м е р 5.1. Пусть оператор $Q^{-1}$ ограничен. Пусть, как в примере 4.1 , множество $G=[0,1]^{d}$, а $\Theta$ состоит из функций $\theta(x)$, периодических с периодом 1 по каждому аргументу. Запишем $\theta(x)$ в виде

$$
\psi\left(e^{2 \pi i x_{1}}, \ldots, e^{2 \pi i x_{d}}\right)=\sum_{j} \theta_{j} e^{2 \pi i(x, j)}
$$

и предположим, что функция $\psi\left(z_{1}, \ldots, z_{d}\right)$ аналитична в области $\left\{1-\alpha_{1}<\left|z_{1}\right|<1+\beta_{1}, \ldots, 1-\alpha_{d}<\left|z_{d}\right|<1+\beta_{d}\right\}$, где $\alpha_{i}>0, \beta_{i}>0$ - заданные числа, и ограничены там некоторой постоянной $C$. Легко видеть, что в этом случае $\Theta$ содержится в некотором эллипсоиде в $L_{2}$ с логарифмами полуосей $\ln a_{n} \leqslant C n^{1 / d}$ и содержит эллипсоид с $\ln a_{n} \geqslant c n^{1 / d}, c>0$. Из доказательства теоремы 5.3 легко следует, что

$$
\Delta_{\varepsilon}(\Theta) \asymp \varepsilon^{p}\left|\ln ^{d p / 2} \frac{1}{\varepsilon}\right| .
$$

II р и м е p 5.2. Рассмотрим следуюшие классы гладких функций, являюшиеся аналогами классов функций из примера 4.1. Допустим, 
что $G$ - ограниченная область в $\mathbf{R}^{d}$ с границей, удовлетворяющей условию конуса (см. [12]). Пусть $R$ - самосопряженный неотрицательный оператор с областью определения $D(R) \subseteq L_{2}(G)$, порожденный формально самосопряженным равномерно эллиптическим оператором $A=\sum_{|j| \leqslant \nu} \alpha_{j} D^{j}$ с постоянными коэффициентами. Пусть $\Theta=\{\theta \in D(R):(R \theta, \theta) \leqslant 1\}$. В работе [13] доказано, что поперечники Колмогорова

$$
d_{n}(\Theta)=\left(\frac{\lambda}{n}\right)^{\nu /(2 d)}(1+o(1))
$$

где

$$
\lambda=(2 \pi)^{-d} \operatorname{mes} G \operatorname{mes}\left\{\xi: 0<\sum \alpha_{j} \xi^{j}<1\right\} .
$$

Поэтому $\Delta_{\varepsilon}(\Theta) \asymp \varepsilon^{2 p \beta /(2 \beta+1)}$, где число $\beta=\nu /(2 d)$.

5.4. Пусть оператор $Q=I$, а множество $\Theta$ есть эллипсоиц $\Theta\left\{\left\{a_{n}\right\}\right\}$. В работе Пинскера [14] для задач оценивания в гауссовском белом шуме была развита теория, позволяющая для эллипсоидальных параметрических множеств устанавливать в ряде случаев точное асимптотическое поведение функций риска $\mathbf{E}_{\theta}\left\|\theta_{\varepsilon}-\theta\right\|^{2}$. Позднее в работах Левита и Голубева (см., например, [15]) были найдены более простые подходы к доказательству результатов Пинскера. Следуя [15], можно установить, что в предположениях этого примера при $\varepsilon \rightarrow 0$

$$
\Delta_{\varepsilon}^{(2)}(\Theta)=\inf _{\theta_{\varepsilon}} \sup _{\theta} \mathbf{E}_{\theta}\left\|\theta_{\varepsilon}-\theta\right\|^{2} \sim \sum_{n=1}^{\infty}\left(1-\varkappa a_{n}\right)_{+},
$$

а число $\varkappa$ определяется из соотношений

$$
\varkappa=\varepsilon^{2} \sum_{n=1}^{\infty} a_{n}\left(1-\varkappa a_{n}\right)_{+} .
$$

В тех случаях, когда $a_{n} \sim a n^{\beta}(\ln n)^{\gamma}$ или $a_{n} \sim c_{1} e^{c_{2} n}$, из (5.7) можно вывести асимптотические формулы вида

$$
\begin{aligned}
& \Delta_{\varepsilon}^{(2)}(\Theta) \sim c(\beta, \gamma, a) \varepsilon^{4 \beta /(2 \beta+1)}\left(\ln \frac{1}{\varepsilon}\right)^{-2 \gamma /(2 \beta+1)}, \\
& \Delta_{\varepsilon}^{(2)}(\Theta) \sim c\left(c_{1}, c_{2}\right) \varepsilon^{2} \ln \frac{1}{\varepsilon} .
\end{aligned}
$$

Точные асимптотические формулы для $\Delta_{\varepsilon}^{p}(\Theta)$ и $p \neq 2$ неизвестны.

Заметим еще, что роль поперечников в задачах оценивания впервые была понята Н.Н. Ченцовым [11]; $\varepsilon$-энтропия, $\varepsilon$-емкость и пропускная способность при решении задач оценивания, по-видимому, впервые были использованы авторами в [17]. 


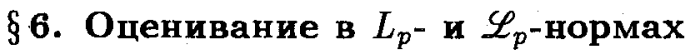

6.1. В этом параграфе мы продолжаем рассматривать примеры оценивания $\theta \in \Theta$, когда $\Theta$ есть класс гладких функций типа рассмотренного в $\S 5$, но когда близость оценки к $\theta$ меряется в $L_{p^{-}}$или

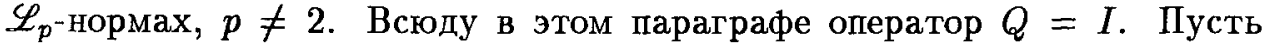
вектор $\beta=\left(\beta_{1}, \ldots, \beta_{n}\right), \beta_{j}=r_{j}+\alpha_{j}$, где $r_{j} \geqslant 0$ - целые числа, а $0<\alpha_{j} \leqslant 1$. Пусть $\Omega-$ область в $\mathbf{R}^{n}$. Обозначим $H_{p}^{\beta} A(\Omega)$ класс функций $f\left(y_{1}, \ldots, y_{n}\right)$ таких, что $f \in L_{p}(\Omega)$, имеет там производные $\partial^{r_{j}} f / \partial y_{j}^{r_{j}}$ и, в случае $p<\infty$, производная $\partial^{r_{j}} f / \partial y_{j}^{r_{j}}$ удовлетворяет неравенству

$$
\begin{aligned}
& \left(\int_{\Omega}\left|f_{y_{j}}^{\left(r_{j}\right)}\left(y_{1}, \ldots, y_{j}+h, \ldots, y_{n}\right)-f_{y_{j}}^{\left(r_{j}\right)}\left(y_{1}, \ldots, y_{n}\right)\right|^{p} d y_{1} \cdots d y_{n}\right)^{1 / p} \\
& \quad \leqslant A|h|^{\alpha_{j}}
\end{aligned}
$$

Если $p=\infty$, последнее неравенство заменяется неравенством

$$
\sup _{x}\left|f_{x_{j}}^{\left(r_{j}\right)}\left(x_{1}, \ldots, x_{j}+h, \ldots, x_{n}\right)-f_{x_{j}}^{\left(r_{j}\right)}\left(x_{1}, \ldots, x_{n}\right)\right| \leqslant A|h|^{\alpha_{j}} .
$$

Нормированное пространство функций на $G$ с нормой

$$
\|f\|=\|f\|_{p}+\sup _{h} \frac{1}{|h|^{\alpha_{0}}}\left\|\Delta_{t h} \frac{\partial^{r_{0}} f}{\partial t^{r_{0}}}\right\|_{p}+\sum_{j=1}^{d} \sup _{h} \frac{1}{|h|^{\alpha_{j}}}\left\|\Delta_{x_{j} h} \frac{\partial^{r_{j}} f}{\partial x_{j}^{r_{j}}}\right\|_{p},
$$

где

$$
\Delta_{x_{j} h} g=g\left(\ldots, x_{j-1}, x_{j}+h, \ldots\right)-g\left(\ldots, x_{j-1}, x_{j}, \ldots\right)
$$

- пространство Никольского (см. [18])- обозначается $H_{p}^{\beta}([0, T] \times G)$.

В этом параграфе мы хотим доказать ряд результатов, которые означают, вообще говоря, что для областей $G$ с гладкой границей и для $\Theta=H_{p}^{\beta}([0, T] \times G) A$

$$
\begin{aligned}
\Delta_{\varepsilon}^{(p)}(\Theta) & =\inf _{\theta_{\varepsilon} \sup _{\theta \in \Theta} \mathbf{E}_{\theta}\left\|\hat{\theta}_{\varepsilon}-\theta\right\|_{p}} \\
& \asymp \begin{cases}\varepsilon^{2 \beta /(2 \beta+1)}, & 2 \leqslant p<\infty, \frac{1}{\beta}=\frac{1}{\beta_{1}}+\cdots+\frac{1}{\beta_{d}}, \\
\varepsilon^{2 \beta /(2 \beta+1)}\left(\ln \frac{1}{\varepsilon}\right)^{\beta /(2 \beta+1)}, & p=\infty .\end{cases}
\end{aligned}
$$

Чтобы упростить конструкцию оценок для $\theta$ и избавиться от трудностей, связанных с оценкой значений $\theta(x)$ для $x$, близких к границе $G$, мы будем на самом деле доказывать неравенства, несколько более слабые. Именно, пусть открытое множество $g \subset \bar{g}_{1} \subset G$, где $g_{1}$ - тоже открытое множество. Вместо $\Delta_{\varepsilon}^{(p)}(\Theta)$ будем оценивать выражение

$$
\Delta_{\varepsilon}^{(p)}(\Theta ; g)=\inf _{\theta_{\varepsilon}} \sup _{\theta} \mathbf{E}_{\theta}\left\|\theta_{\varepsilon}-\theta \mid L_{p}((0, T) \times g)\right\|
$$


причем всякий раз, когда это не может вызвать недоразумения, мы пишем $|\cdot|_{p},\|\cdot\|_{p}$ вместо $\left\|\cdot\left|L_{p}(g)\|,\| \cdot\right| L_{p}((0, T) \times g)\right\|$.

Теорема 6.1. Пусть $g, g_{1}$ - два открытых множества и $g \subset$ $\bar{g}_{1} \subset G$. Пусть $\Theta=H_{p}^{\beta}([0, T] \times G) A$. Тогда

$$
\Delta_{\varepsilon}^{(p)}(\Theta ; g) \asymp \begin{cases}\varepsilon^{2 \beta /(2 \beta+1)}, & 2 \leqslant p<\infty \\ \varepsilon^{2 \beta /(2 \beta+1)}\left(\ln \frac{1}{\varepsilon}\right)^{\beta /(2 \beta+1)}, & p=\infty\end{cases}
$$

В этом пункте мы построим оценки $\theta_{\varepsilon}$, на которых достигается $(6.3)$; в следующем I. 6.2 будут указаны границы снизу для $\Delta_{\varepsilon}$.

Можно предложить следующий обший метод построения оценок $\theta_{\varepsilon}$. Пусть $A_{\delta}: \mathscr{L}_{2} \rightarrow \mathscr{L}_{2}, \delta>0,-$ семейство операторов Гильберта-Шмидта такое, что

$$
\sup _{\theta}\left\|A_{\delta} \theta-\theta\right\|_{p} \underset{\delta \rightarrow 0}{\longrightarrow} 0 .
$$

Тогда оценки $A_{\delta}\left(\dot{u}_{\varepsilon}-L u_{\varepsilon}\right)$ будут состоятельными оценками для $A_{\delta} \theta$ при фиксированном $\delta>0$ (см. $\S 2$ ), и, следовательно, при подходяшем выборе $\delta=\delta(\varepsilon)$ оценки $\theta_{\varepsilon}=A_{\delta(\varepsilon)}\left(\dot{u}_{\varepsilon}-L u_{\varepsilon}\right)$ будут состоятельными оценками для $\theta$. Остается оптимальным образом подобрать семейство $\left\{A_{\delta}\right\}$. Оператор $A_{\delta}$ - интегральный и допускает выражение

$$
A_{\delta} \theta(t, x)=\int_{0}^{T} \int_{G} a_{\delta}(t, x ; s, y) \theta(s, y) d y d s
$$

Поэтому оценки $A_{\delta}\left(\dot{u}_{\varepsilon}-L u_{\varepsilon}\right)$ будут ядерными оценками,

$$
A_{\delta}\left(\dot{u}_{\varepsilon}-L u_{\varepsilon}\right)(t, x)=\int_{0}^{T}\left\langle a_{\delta}(t, x ; s, \cdot), d u_{\varepsilon}(t)-L u_{\varepsilon}(t) d t\right\rangle
$$

Очевидно,

$$
A_{\delta}\left(\dot{u}_{\varepsilon}-L u_{\varepsilon}\right)(t, x)=\int_{0}^{T} \int_{G} a_{\delta}(t, x ; s, y) \theta(s, y) d y d s+\varepsilon \int_{0}^{T}\left\langle a_{\delta}, d w\right\rangle
$$

Поэтому величина, например, среднеквадратической погрешности $\mathrm{E}_{\theta}\left|\theta_{\varepsilon}-\theta\right|^{2}$ складывается из погрешности аппроксимации

$$
\left|\theta(t, x)-\int_{0}^{T} \int_{G} a_{\delta}(t, x ; s, y) \theta(s, y) d y d s\right|^{2}
$$

функции $\theta(t, x)$ соответствующим интегралом и из цисперсии

$$
\varepsilon^{2} \mathbf{E}\left|\int_{0}^{T}\left\langle a_{\delta}, d w\right\rangle\right|^{2}=\varepsilon^{2} \int_{0}^{T} \int_{G} a_{\delta}^{2}(t, x ; s, y) d y d s
$$

и нужно построить ядра $a_{\delta}$, минимизируюшие сумму (6.4) и (6.5). Распространенный выбор ядер $a$ для аппроксимации гладких функций 
$\theta(t, x)$ состоит в следуюшем. Пусть $\varphi(y)-$ функция вепественной переменной $y$ такая, что

$$
\begin{gathered}
\int_{-\infty}^{\infty} \varphi(y) d y=1, \quad \int_{-\infty}^{\infty} y^{j} \varphi(y) d y=0, \quad j=1, \ldots, m, \\
\int_{-\infty}^{\infty}\left(|y|^{m+1}+1\right)|\varphi(y)| d y<\infty .
\end{gathered}
$$

Задавшись вектором $\delta=\left(\delta_{0}, \delta_{1}, \ldots, \delta_{d}\right)$, определим ядра $a_{\delta}$ равенством

$$
a_{\delta}(t, x ; s, y)=\left(\delta_{0} \cdots \delta_{d}\right)^{-1} \varphi\left(\delta_{0}^{-1}(t-s)\right) \prod_{1}^{d} \varphi\left(\delta_{j}^{-1}\left(x_{j}-y_{j}\right)\right) .
$$

Свертки ядер $a_{\delta} * \theta$ хорошо приближают $\theta$.

Лемма 6.1. Если $\theta \in H_{p}^{\beta}\left(\mathbf{R}^{d+1}\right) A$, mo

$$
\left\|a_{\delta} * \theta-\theta\right\|_{p} \leqslant C(A, p) \sum_{0}^{d} \delta_{j}^{\beta_{j}} .
$$

Д о к а 3 а т е л в с т в о леммы мало отличается от доказательства теоремы 5.2.4 из [18], и мы его опустим, тем более что ниже нам будет достаточно этой теоремы.

Применение леммы 6.1 к оценке погрешности (6.4) наталкивается на ту трудность, что область $G$ не совпадает со всем пространством и свертки $a_{\delta} * \theta$, вообще говоря, не будут определены. Можно предложить цва способа, позволяюпих избежать этого затруднения.

Первый способ состоит в следующем. Пусть функция $\varphi(y)$ в (6.6) финитна и, скажем, $\varphi(y)=0$ для $|y| \geqslant 1$. Тогда свертка $a_{\delta} * \theta$ определена для всех $(t, x)$, удаленных от границы $[0, T] \times G$ на величину порядка $|\delta|$. В частности, при малых $|\delta|$ свертка $a_{\delta} * \theta$ будет определена для всех $x \in g$, где $g-$ множество из теоремы 6.1 .

Второй способ связан с продолжением функций за пределы множества $G$. Именно, пусть $R$ - линейный ограниченный оператор одновременно из $H_{p}^{\beta}([0, T] \times G)$ в $H_{p}^{\beta}\left(\mathbf{R}^{d+1}\right)$ и из $L_{2}([0, T] \times G)$ в $L_{2}\left(\mathbf{R}^{d+1}\right)$. Обозначим $R^{*}$ оператор, сопряженный $R$. Свертка $\left[R^{*} a_{\delta}\right] * \theta$ корректно определена, и по лемме 6.1

$$
\left\|\left(R^{*} a_{\delta}\right) * \theta-\theta\right\|_{p}=\left\|a_{\delta} *(R \theta)-R \theta\right\|_{p} \leqslant C \sum \delta_{j}^{\beta_{j}} .
$$

В то же время в силу (6.5)

$$
\begin{aligned}
\mathbf{E}\left|\varepsilon \int_{0}^{T}\left\langle R^{*} a_{\delta}, d w\right\rangle\right|^{2} & =\varepsilon^{2} \int_{0}^{T} \int_{G}\left|R^{*} a_{\delta}\right|_{2}^{2} d y d s \\
& \leqslant \varepsilon^{2}\|R\|^{2} \int_{\mathbf{R}^{d+1}}\left|a_{\delta}\right|^{2} d s d y \leqslant C \varepsilon^{2} \delta_{0}^{-1} \cdots \delta_{d}^{-1} .
\end{aligned}
$$


Поэтому если такой оператор $R$ существует, то, полагая $\delta_{j}=$ $\varepsilon^{2 \beta_{j} /\left(2 \beta_{j}+1\right)}$, мы найдем, например, из (6.7) и (6.8) для $p=2$ и оценки

$$
\theta_{\varepsilon}=\int_{0}^{T}\left\langle R^{*} a_{\delta}, d u_{\varepsilon}(t)-L u_{\varepsilon}(t) d t\right\rangle
$$

что

$$
\mathbf{E}\left\|\theta_{\varepsilon}-\theta\right\| \leqslant C \varepsilon^{2 \beta /(2 \beta+1)} .
$$

Величина постоянной $C$ в неравенстве (6.9) при реальных расчетах, конечно, важна. Выбор ядра $a_{\delta}$, на котором $C$ достигает значения, близкого к минимальному, может зависеть от области $G$. Поскольку в настоящей работе мы ограничиваемся разысканием опенок, оптимальных лишь по порядку скорости сходимости функций риска к нулю при $\varepsilon \rightarrow 0$, оставляя в стороне важную задачу исследования постоянных, мы предпочитаем иметь дело с универсальными ядрами $a_{\delta}$. При этом, решая задачу приближения $\theta$ свертками $a_{\delta} * \theta$, мы будем опираться на готовые результаты теории приближений [18]. Речь идет о приближении целыми функциями конечной степени в пространствах $L_{p}\left(\mathbf{R}^{d}\right), 2 \leqslant p \leqslant \infty$.

Напомним, что функция $g\left(z_{1}, \ldots, z_{n}\right)$ есть целая функция степени $\nu=\left(\nu_{1}, \ldots, \nu_{n}\right)$, если она есть целая функция по всем переменным и если для всякого $\varepsilon>0$ существует число $A_{\varepsilon}>0$ такое, что

$$
|g(z)| \leqslant A_{\varepsilon} \exp \left\{\sum_{1}^{n}\left(\nu_{j}+\varepsilon\right)\left|z_{j}\right|\right\}
$$

Лемма 6.2 ([18, с. 150]). Если $1 \leqslant p<q \leqslant \infty$, то для иелой функции $g \in L_{p}\left(\mathbf{R}^{n}\right)$ конечной степени $\nu=\left(\nu_{1}, \ldots, \nu_{n}\right)$ имеет место неравенст60

$$
\|g\|_{q} \leqslant 2^{n}\left(\nu_{1} \cdots \nu_{n}\right)^{1 / p-1 / q}\|g\|_{p}
$$

Обозначим $E_{\nu}^{(p)}(f)$ величину наилучшего приближения функции $f \in L_{p}\left(\mathbf{R}^{n}\right)$ целыми функциями $g_{\nu}$ степени $\nu: E_{\nu}^{(p)}(f)=\inf _{g_{\nu}}\left\|f-g_{\nu}\right\|_{p}$. Tozda

Лемма $6.3([18$, c. 226$])$. Пусть $f \in H_{p}^{\beta}\left(\mathbf{R}^{n}\right) A, \beta=\left(\beta_{1}, \ldots, \beta_{n}\right)$.

$$
E_{\nu}^{(p)}(f) \leqslant C \sum_{1}^{n} \nu_{j}^{-\beta_{j}}
$$

Положим

$$
V_{N}(t)=\frac{\cos N t-\cos 2 N t}{\pi N t^{2}}, \quad t \in \mathbf{R}^{1} .
$$

Определим ядра Валле-Пуссена $V_{\nu}(x), \nu=\left(\nu_{0}, \ldots, \nu_{d}\right)$, равенством

$$
V_{\nu}(t, x)=V_{\nu_{0}}(t) \prod_{1}^{d} V_{\nu_{j}}\left(x_{j}\right) .
$$

Очевидно, $V_{\nu}$ и свертки $V_{\nu} * f$ суть целые функции степени $2 N$. 
Лемма 6.4 ([18, с. 350]). Имеет место неравенство

$$
\left\|\int_{\mathbf{R}^{n}} V_{\nu}(x-y) f(y) d y-f\right\|_{p} \leqslant(1+M) E_{\nu}^{(p)}(f),
$$

аде $M$ - постоянная, зависящая только от размерности $n$.

Переходя к построению оценок $\theta_{\varepsilon}$ и следуя указанной выше схеме, определим операторы продолжения $R$ и выберем в качестве ядер $a_{\delta}$ ядра $R^{*} V_{\nu}$. Существуют различные конструкции операторов продолжения (см., например, [20, гл. VI], [21]); для доказательства теоремы 6.1, в которой функции риска суть нормы уклонения оценок от $\theta$ в $L_{p}(g)$ и $g \subset \bar{g}_{1} \subset G$, такие операторы продолжения можно построить совсем просто (см. [18, с. 447]). Именно, пусть $\varphi(x)$ - бесконечно дифференцируемая на $\mathbf{R}^{d}$ функция такая, что $\varphi(x)=1$ на $g$ и $\varphi(x)=0$ вне $G$. Определим оператор $R_{1}$, продолжающий функции, определенные на $g$, до функций, определенных на $\mathbf{R}^{d}$, с помощью равенства $R_{1} f(x)=\varphi(x) f(x)$. Очевидно, $R_{1}$ есть линейный ограниченный оператор и как оператор из $H_{p}^{\beta}(g)$ в $H_{p}^{\beta}\left(\mathbf{R}^{d}\right)$ и как оператор из $L_{2}(g)$ в $L_{2}\left(\mathbf{R}^{d}\right)$. Далтее, если $f(t, x)$ есть функция, определенная на $[0, T] \times \mathbf{R}^{d}$, то она может быть продолжена без труда до функции на $(-\infty, \infty) \times \mathbf{R}^{d}=\mathbf{R}^{d+1}$ с помощью конструкции Хестенса, оператор продолжения $R_{2}$; например, для малых $|t|$ и $t<0$ продолженная функция $\bar{f}(t, x)=\sum_{s=1}^{k} c_{s} f\left(-t \cdot 2^{-s}, x\right)$ при подходяпем подборе чисел $k, c_{s}$ (см. [20, с. 231], и цитируемую там литературу). Операторы продолжения $R_{2}$ - линейные ограниченные операторы в $H_{p}^{\beta}([0, T] \times g)$ и в $L_{2}([0, T] \times g)$, и мы можем, наконец, огределить искомые операторы продолжения $R=R_{2} R_{1}$.

Определим теперь оценки

$$
\theta_{\nu}(t, x)=\int_{0}^{T}\left\langle R^{*} V_{\nu}(x-\cdot), d u_{\varepsilon}(t)-L u_{\varepsilon}(s)\right\rangle
$$

$\nu=\left(\nu_{0}, \ldots, \nu_{d}\right)$, функции $\theta(t, x)$ и исследуем их свойства.

Лемма 6.5. Имеют место неравенства

$$
\begin{aligned}
\mathbf{E}\left\|\theta_{\nu}-\theta\right\|_{p} & \leqslant C\left(E_{\nu}^{(p)}(\theta)+\varepsilon\left(\nu_{0} \cdots \nu_{d}\right)^{1 / 2}\right), \quad 2 \leqslant p<\infty \\
\mathbf{E}\left\|\theta_{\nu}-\theta\right\|_{\infty} & \leqslant C\left(E_{\nu}^{(\infty)}(\theta)+\varepsilon\left(\nu_{0} \cdots \nu_{d}\right)^{1 / 2} \ln \left(\nu_{0} \cdots \nu_{d}\right)\right)
\end{aligned}
$$

Постоянные $C$ зависят от $p$ и областей $g, g_{1}, G$.

Д ок азатель с т в о. Очевидно,

$$
\begin{aligned}
\theta_{\nu}(t, x)-\theta(t, \dot{x})= & {\left[\int_{0}^{T}\left\langle R^{*} V_{\nu}(t-s, x-y), \theta(s, y)\right\rangle d s-\theta(t, x)\right] } \\
& +\varepsilon \int_{0}^{T}\left\langle R^{*} V_{\nu}(t-s, x-y), d w(s)\right\rangle .
\end{aligned}
$$


Поэтому

$$
\mathbf{E}\left\|\theta_{\nu}-\theta\right\|_{p} \leqslant\left\|\int_{0}^{T}\left\langle R^{*} V_{\nu}, \theta\right)-\theta\right\|_{p}+\varepsilon \mathbf{E}\left\|\int_{0}^{T}\left\langle R^{*} V_{\nu}, d w(s)\right\rangle\right\|_{p} .
$$

Первое слагаемое в (6.14) легко оценивается с помощью лемм 6.3, 6.4. Именно, в силу (6.12)

$$
\left\|\int_{0}^{T}\left\langle R^{*} V_{\nu}, \theta\right\rangle d s-\theta\right\|_{p} \leqslant\left\|V_{\nu} * R \theta-R \theta \mid L_{p}\left(\mathbf{R}^{d+1}\right)\right\| \leqslant(1+M) E_{\nu}^{(p)}(R \theta) .
$$

В силу же (6.11) для $\theta \in H_{p}^{\beta}([0, T] \times G) A$ отсюда следует, что

$$
\left\|\int_{0}^{T}\left\langle R^{*} V_{\nu}, \theta\right\rangle d s-\theta\right\|_{p} \leqslant C \sum_{0}^{d} \nu_{j}^{-\beta_{j}} .
$$

Осталось оценить второе слагаемое в (6.14), норму случайной функции

$$
\xi(t, x)=\int_{0}^{T}\left\langle R^{*} V_{\nu}, d w(s)\right\rangle
$$

Это гауссовская функция с $\mathbf{E} \xi^{2}(t, x) \leqslant\left\|R^{*}\right\|_{2}^{2}\left\|V_{\nu}\right\|^{2}=C\|R\|_{2}^{2} \nu_{0} \cdots \nu_{d}$. Поэтому

$$
\begin{aligned}
\mathbf{E}|\xi(t, x)|^{p} & =\left(\mathbf{E} \xi^{2}(t, x)\right)^{p / 2} \frac{1}{\sqrt{2 \pi}} \int_{-\infty}^{\infty}|x|^{p} e^{-x^{2} / 2} d x \\
& \leqslant C^{p}\|R\|_{2}^{p} p^{p / 2}\left(\nu_{0} \cdots \nu_{d}\right)^{p / 2} .
\end{aligned}
$$

Следовательно, для $p \leqslant 2<\infty$ (множество $g$ ограничено)

$$
\mathbf{E}\|\xi\|_{p} \leqslant \mathbf{E}^{1 / p}\|\xi\|_{p}^{p} \leqslant C\|R\|_{2 \sqrt{p}\left(\nu_{0} \cdots \nu_{d}\right)^{1 / 2} .}
$$

Чтобы оценить $\mathbf{E}\|\xi\|_{\infty}$, заметим сперва, что из неравенств для $\mathbf{E}\|\xi\|_{p}$ легко следует неравенство

$$
\mathbf{E}\|\xi\|_{p} \leqslant C\|R\|_{2} \mathbf{E}\left\|\int_{0}^{T}\left\langle V_{\nu}, d w(s)\right\rangle\right\|_{p}
$$

Функция $\eta(t, x)=\int_{0}^{T}\left\langle V_{\nu}, d w(s)\right\rangle$ есть целая функция степени $2 \nu$. Выберем и зафиксируем малое положительное число $\delta>0$ так, чтобы для $(t, x) \in[0, T] \times g$

$$
\frac{1}{2} \leqslant \frac{\sin \delta t}{\delta t} \prod_{1}^{d} \frac{\sin \delta x_{j}}{\delta x_{j}} \leqslant 1
$$

Тогда функция

$$
\eta_{\delta}(t, x)=\eta(t, x) \cdot \frac{\sin \delta t}{\delta t} \prod_{1}^{d} \frac{\sin \delta x_{j}}{\delta x_{j}}
$$

есть целая функция степени $\left(2 \nu_{0}+\delta, \ldots, 2 \nu_{d}+\delta\right)$. Ниже $\nu_{j}-$ большие числа, и можно считать, что $\eta_{\delta}$ есть функция степени $3 \nu$. 
По лемме 6.2 для $q>$ ?

$$
\begin{aligned}
\mathbf{E}\|\xi\|_{q} & \leqslant C\|R\|_{2} \mathbf{E}\|\eta\|_{q} \leqslant C 2^{d+1}\|R\|_{2} \mathbf{E}\left\|\eta_{\delta}\right\|_{q} \leqslant C 2^{d+1}\|R\|_{2} \mathbf{E}\left\|\eta_{\delta} \mid \mathbf{R}^{d+1}\right\|_{q} \\
& \leqslant C 2^{d+1}\|R\|_{2}\left(3 \nu_{0} \cdot 3 \nu_{1} \cdots 3 \nu_{d}\right)^{1 / p-1 / q} \mathbf{E}\left\|\eta_{\delta} \mid \mathbf{R}^{d+1}\right\|_{p} \\
& \leqslant C\left(\nu_{0} \cdots \nu_{d}\right)^{1 / 2+1 / p} \sqrt{p} .
\end{aligned}
$$

Следовательно, для всех $q \geqslant 2$

$$
\begin{aligned}
\mathbf{E}\|\xi\|_{q} & \leqslant C\left(\nu_{0} \cdots \nu_{d}\right)^{1 / 2} \min _{p \leqslant q}\left(\sqrt{q},\left(\nu_{0} \cdots \nu_{d}\right)^{1 / p} \sqrt{p}\right) \\
& \leqslant C\left(\nu_{0} \cdots \nu_{d}\right)^{1 / 2}\left(\ln \left(\nu_{0} \cdots \nu_{d}\right)\right)^{1 / 2}
\end{aligned}
$$

По лемме Фату

$$
\mathbf{E}\|\xi\|_{\infty}=\mathbf{E}\left(\lim _{p \rightarrow \infty}\|\xi\|_{p}\right) \leqslant \lim _{p \rightarrow \infty} \mathbf{E}\|\xi\|_{p} \leqslant C\left(\nu_{0} \cdots \nu_{d}\right)^{1 / 2}\left(\ln \left(\nu_{0} \cdots \nu_{d}\right)\right)^{1 / 2} .
$$

Лемма 6.5 доказана. Утверждение теоремы 6.1, касающееся верхних границ, немедленно следует из неравенства леммы 6.5. Действительно, в условиях теоремы $E_{\nu}^{(p)}(\theta) \leqslant C \sum_{0}^{d} \nu_{j}^{-\beta_{j}}$, и неравенства леммы 6.5 принимают вид

$$
\begin{gathered}
\mathbf{E}\left\|\theta_{\nu}-\theta\right\|_{p} \leqslant C\left(\sum_{0}^{d} \nu_{j}^{-\beta_{j}}+\varepsilon\left(\nu_{0} \cdots \nu_{d}\right)^{1 / 2}\right), \quad 2 \leqslant p<\infty, \\
\mathbf{E}\left\|\theta_{\nu}-\theta\right\|_{\infty} \leqslant C\left(\sum_{0}^{d} \nu_{j}^{-\beta_{j}}+\varepsilon\left(\nu_{0} \cdots \nu_{d}\right)^{1 / 2}\left(\ln \left(\nu_{0} \cdots \nu_{d}\right)\right)^{1 / 2}\right),
\end{gathered}
$$

и в качестве оценки $\theta_{\varepsilon}$ можно выбрать оценку $\theta_{\nu}$, где $\nu=\nu(\varepsilon)$ определя्रется из соотношений

$$
\begin{aligned}
& \nu_{j} \asymp \varepsilon^{-2 \beta /\left[\beta_{j}(2 \beta+1)\right]}, \quad p<\infty, \\
& \nu_{j} \asymp \varepsilon^{-2 \beta /\left[\beta_{j}(2 \beta+1)\right]}\left(\ln \frac{1}{\varepsilon}\right)^{-\beta /\left[\beta_{j}(2 \beta+1)\right]}, \quad p=\infty .
\end{aligned}
$$

Теорема 6.1 в отношении верхних границ доказана. Точнее, мы доказали следуюший результат.

Теорема 6.2. Пусть $\Theta \subseteq H_{p}^{\beta}([0, T] \times G) A$. Пусть $g$ - ограниченное открытое множество, $g_{1}$ - открытое множество, и пусть $g \subset \bar{g}_{1} \subset G$. Построенные выше ядерные оченки $\theta_{\varepsilon}$ удовлетворяют неравенствам

$$
\begin{aligned}
& \sup _{\theta} \mathrm{E}_{\theta}\left\|\theta_{\varepsilon}-\theta \mid L_{p}([0, T] \times g)\right\|_{p} \leqslant C(\operatorname{mes} g \cdot T)^{1 / p} \varepsilon^{2 \beta /(2 \beta+1)}, \quad 2 \leqslant p<\infty, \\
& \sup _{\theta} \mathrm{E}_{\theta}\left\|\theta_{\varepsilon}-\theta \mid L_{p}([0, T] \times g)\right\|_{\infty} \leqslant C \varepsilon^{2 \beta /(2 \beta+1)}\left(\ln \frac{1}{\varepsilon}\right)^{\beta /(2 \beta+1)},
\end{aligned}
$$

где постоянные $C$ зависят от $A, \beta, p$ и оператора продолжения $R$. 
3 ам е ч а н и е 6.1. Основную роль в вычислении верхних границ точности оценивания играют неравенства леммы 6.5. Поскольку в этих неравенствах точность аппроксимации $\theta$ оценками $\theta_{\nu}$ определяется величинами наилучших приближений, ясно, что вместо предположения, что $\Theta$ есть подмножество пространства Никольского $H_{p}^{\beta}$, можно было бы предположить, что $\Theta$ есть подмножество какого-либо пространства Соболева, или Бесова, или Лиувилля и т.д., для которых существуют хорошие ощенки величины наилучшего приближения целыми функциями (см. [18]). Вообще же в неравенствах леммы 6.5 величина $E_{\nu}^{(p)}(\theta)$ играет ту же роль, что и поперечники Бернштейна в $\S 5$.

3 а м е ч а н и е 6.2. Предположим, что функция $\theta=\theta(x)$ зависит только. от $x$. Естественно в этом случае вместо оценок $\theta_{\nu}$ рассмотреть оценки

$$
\tilde{\theta}_{\nu}(x)=\frac{1}{T} \int_{0}^{T}\left\langle R_{1}^{*} V_{\nu}(x-\cdot), d u_{\varepsilon}(s)-L u_{\varepsilon}(s) d s\right\rangle,
$$

где теперь $\nu=\left(\nu_{1}, \ldots, \nu_{d}\right)$. В этом случае дисперсия $\mathbf{E}_{\theta}\left|\tilde{\theta}_{\nu}(x)-\mathbf{E} \tilde{\theta}_{\nu}(x)\right|^{2}$ убывает как $\varepsilon^{2} T^{-1}$, и мы приходим к следуюшему результату.

Теорема 6.3. Пусть в условиях теоремь 6.1 функции $\theta=\theta(x)$ зависят только от $x$. Тогда можно подобрать $\nu=\nu(\varepsilon, T)$ так, что для оченок $\theta_{\varepsilon}=\theta_{\nu}$ выполняются неравенства

$$
\begin{aligned}
& \sup _{\theta} \mathbf{E}_{\theta}\left\|\theta_{\varepsilon}-\theta\right\|_{p} \leqslant C\left(\frac{\varepsilon}{\sqrt{T}}\right)^{2 \beta /(2 \beta+1)}, \quad 2 \leqslant p<\infty, \\
& \sup _{\theta} \mathbf{E}_{\theta}\left\|\theta_{\varepsilon}-\theta\right\|_{\infty} \leqslant C\left(\frac{\varepsilon}{\sqrt{T}}\right)^{2 \beta /(2 \beta+1)}\left(\ln \frac{\sqrt{T}}{\varepsilon}\right)^{\beta /(2 \beta+1)} .
\end{aligned}
$$

3 а м е ч а н и е 6.3. Рассмотренные выше ядерные оценки решают также задачу оценивания значения $\theta(t, x)$ в точке $(t, x)$. При этом, конечно, нет необходимости вводить операторы продолжения: Достаточно рассмотреть оценки с ядрами типа (6.6), (6.7), равными $\Phi_{\nu}=\left(\nu_{0} \cdots \nu_{d}\right) \varphi\left(\nu_{0}(t-s)\right) \prod_{1}^{d} \varphi\left(\nu_{j}\left(x_{j}-y_{j}\right)\right)$, где дополнительно предполагается, что $\varphi-$ финитная функция. При больших $\nu_{j}$ оценки

$$
\theta_{\nu}(x)=\int_{0}^{T}\left\langle\Phi_{\nu}, d u_{\varepsilon}(s)-L u_{\varepsilon}(s) d s\right\rangle
$$

корректно определены. Проведенные выше рассуждения позволяют доказать следующий результат:

если все $\theta \in H_{\infty}^{\beta}(\Lambda) A$, где $\Lambda-$ какая-нибудь окрестность точки $x$, то для $\theta_{\varepsilon}=\theta_{\nu(\varepsilon)}(x), \nu_{j}(\varepsilon) \asymp \varepsilon^{-2 \beta /\left(\beta_{j}(2 \beta+1)\right)}$,

$$
\sup _{\theta} \mathrm{E}_{\theta}\left|\theta_{\varepsilon}-\theta(x)\right|^{r} \leqslant C_{r} \varepsilon^{2 \beta r /(2 \beta+1)} .
$$


6.2. В этом пункте мы приведем нижние границы точности оценивания в метриках $L_{p}$.

Теорема 6.4. Пусть $g \subset G$-открытое множество. Если для каких-нибудь $\beta, A H_{p}^{\beta}([0, T] \times g) A \subseteq \Theta$, mo

$$
\Delta_{\varepsilon}^{(p)}(\Theta) \geqslant \begin{cases}c \varepsilon^{2 \beta /(2 \beta+1)}, & 2 \leqslant p<\infty, \\ c \varepsilon^{2 \beta /(2 \beta+1)}\left(\ln \frac{1}{\varepsilon}\right)^{\beta /(2 \beta+1)}, & p=\infty,\end{cases}
$$

где, как и.өыше, $\beta^{-1}=\sum_{0}^{d} \beta_{j}^{-1}$, ас - положительные постоянные.

Д о к аз а т е л ь с т в о. Мы будем опираться на неравенство (4.6). Без потери общности можно считать, что $g$ есть куб $[0,1]^{d}$ и что $T=1$, $A=1$. Пусть $\varphi(y) \geqslant 0$ есть бесконечно дифференцируемая функция, равная нулю вне отрезка $[0,1]$. Пусть $N=N(\varepsilon)$ - большое целое число, окончательный выбор которого мы уточним позднее. Пусть $\nu_{0}, \ldots, \nu_{d}-$ целые числа, ближайшие к решению экстремальной задачи

$$
z_{0}^{-\beta_{0}}+\cdots+z_{d}^{-\beta_{d}} \longrightarrow \min , \quad z_{0} \cdots z_{d}=N, \quad z_{j}>0 .
$$

Ясно, что $\nu_{j} \asymp N^{\beta / \beta_{j}}$.

Определим функции $\psi_{i}(t, x), i=\left(i_{0}, \ldots, i_{d}\right), 0 \leqslant i_{j} \leqslant \nu_{j}$, равенствами

$$
\psi_{i}(t, x)=N^{-\beta} \varphi\left(\nu_{0}\left(t-\frac{i_{0}}{\nu_{0}}\right)\right) \prod_{j=1}^{d} \varphi\left(\nu_{j}\left(x_{j}-\frac{i_{j}}{\nu_{j}}\right)\right) .
$$

Очевидно, при разных $k, l$ множества $\left\{(t, x): \psi_{k}(t, x)=0\right\}$ и $\{(t, x)$ : $\left.\psi_{l}(t, x)=0\right\}$ не имеют общих точек. Определим, наконеш, функции $f_{a}$ равенствами

$$
f_{a}(t, x)=\sum_{i} a(i) \psi_{i}(t, x),
$$

где $a(i)$ принимает значения 0,1 , а суммирование ведется по всем векторам $i$.

Очевидно, $\mathscr{L}_{2}$-норма $f_{a}$

$$
\left\|f_{a}\right\|=N^{-\beta}\left(\nu_{0} \cdots \nu_{d}\right)^{-1 / 2}\left(\sum_{i} a^{2}(i)\right)^{1 / 2}\|\varphi\|_{2}^{d+1} \leqslant c N^{-\beta}=r,
$$

так что все функции $f_{a}$ принадлежат $\mathscr{L}_{2}$-шару $O_{\tau}$ радиуса $r$.

Далее, легко видеть, что функцию $\varphi$ можно выбрать так, чтобы все функции $f_{a} \subseteq H_{p}^{\beta}([0, T] \times g) \subseteq \Theta$ для нужного $p, 2 \leqslant p \leqslant \infty$. Поэтому если мы обозначим $F$ совокупность всех $f_{a}$, то $F \subseteq \Theta \cap O_{r} \subseteq \Theta$ и в силу (4.6)

$$
\Delta_{\varepsilon}^{(p)}(\Theta)=\inf _{\theta_{\varepsilon}} \sup _{\theta \in F} \mathbf{E}_{\theta}\left\|\theta_{\varepsilon}-\theta\right\|_{p} \geqslant \sup _{\delta} \delta\left(1-\frac{\mathscr{C}_{\varepsilon}(F)+1}{C_{2 \delta}^{(p)}(F)-1}\right),
$$

где $C_{2 \delta}^{(p)}(F)$ означает $2 \delta$-емкость множества $F$ в $\mathscr{L}_{p}$. Дальнейшие рассуждения для случаев $p<\infty$ и $p=\infty$ несколько различаются. 
Пусть сперва $p<\infty$. В силу (4.3)

$$
\mathscr{C}_{\varepsilon}(F) \leqslant \mathscr{C}_{\varepsilon}\left(O_{r}\right) \leqslant \frac{1}{2 \varepsilon^{2} r^{2}}
$$

Обозначим $F_{1} \subseteq F$ множество тех $f_{a}$, для которых если $f_{a}, f_{a^{\prime}} \in F_{1}$, то

$$
\sum_{i}\left|a(i)-a^{\prime}(i)\right|>\frac{1}{4}\left(\nu_{0}-1\right) \cdots\left(\nu_{d}-1\right) .
$$

Лемма 6.6. Мощность множества $F_{1}$

$$
\operatorname{card}\left(F_{1}\right) \geqslant \exp \left\{\frac{1}{8}\left(\nu_{0}-1\right) \cdots\left(\nu_{d}-1\right)\right\} .
$$

Эта лемма в свою очередь вытекает из следующего результата, доказательство которого можно найти в [22] - лемма 7 этой работы (в [22] ошибочно указано, что функшия $a(i)$ принимает значения \pm 1 , а не 0,1$)$.

Лемма 6.7. Рассмотрим Функчию $a(\omega)$, определенную на конечном множестве $\Omega=\{\omega\}, \operatorname{card} \Omega=R$, и принимаюшую значения 0,1 . Обозначим $D$ множество тех функиий $a$, при любьх $a, a^{\prime} \in D$

$$
\sum_{\omega}\left|a(\omega)-a^{\prime}(\omega)\right| \geqslant \frac{R}{4} \text {. }
$$

Tozda

$$
\operatorname{card} D \geqslant\left[2^{-R} \sum_{k=0}^{[R / 4]} C_{R}^{k}\right]^{-1} \geqslant \exp \left\{\frac{R}{8}\right\}
$$

Заметим, что первая часть последнего неравенства есть неравенство Гильберта или Варшамова-Гильберта из теории кодирования, см. [23]. В торая же легко следует из элементарных теорем о больших уклонениях для испытаний Бернулли.

Пусть теперь $f_{a}, f_{a^{\prime}}$ - какие-либо две функпии из $F_{1}$. Тогда

$$
\begin{aligned}
\left|f_{a}-f_{a^{\prime}}\right|_{p} & =\left(\sum_{i}\left|a(i)-a^{\prime}(i)\right| \int\left|\psi_{i}(t, x)\right|^{p} d t d x\right)^{1 / p} \\
& =N^{-\beta}\left(\nu_{0} \cdots \nu_{d}\right)^{-1 / p}\|\varphi\|_{p}^{d+1}\left(\sum_{i}\left|a(i)-a^{\prime}(i)\right|\right)^{1 / p} \\
& \geqslant 8^{-p}\|\varphi\|_{p}^{d+1} N^{-\beta}=c N^{-\beta}
\end{aligned}
$$

где постоянная $c>0$. Обозначая правую часть последнего неравенства $2 \delta$, найдем, что $F_{1}$ состоит из $2 \delta$-различимых точек. При таком выборе $\delta$

$$
\frac{\mathscr{C}_{\varepsilon}(F)-1}{C_{2 \delta}^{(p)}(F)+1} \leqslant c \frac{r^{2}}{\varepsilon^{2} \ln \operatorname{card}\left(F_{1}\right)} \leqslant c \varepsilon^{-2} N^{-2 \beta-1} \leqslant \frac{1}{2},
$$

если вдобавок $N$ выбрано так, что $N \sim(4 c)^{1 /(2 \beta+1)} \varepsilon^{-2 /(2 \beta+1)}$. В силу $(6.16) \Delta_{\varepsilon}^{(p)}(\Theta) \geqslant c \varepsilon^{2 \beta /(2 \beta+1)}$. 
Пусть теперь $p=\infty$. Обозначим $F_{2}$ множество $f_{a}$, отвечающих таким функциям $a$, что ровно одно из значений $a(i)$ равно 1 , а остальные суть нули. Очевидно, $\operatorname{card}\left(F_{2}\right)=\nu_{0} \cdots \nu_{d} \sim N$. Далее, если $f_{a}, f_{a^{\prime}} \in F_{2}$, то

$$
\left|f_{a}-f_{a^{\prime}}\right|_{\infty}=N^{-\beta}\|\varphi\|_{\infty}^{d+1} \text {. }
$$

Наконец, если $f_{a} \in F_{2}$, то $\left\|f_{a}\right\|=N^{-\beta-1 / 2}\|\varphi\|_{2}^{d+1}$. Поэтому, полагая $2 \delta=N^{-\beta}\|\varphi\|_{\infty}^{d+1}$ и выбирая $N \sim c \varepsilon^{-2 /(1+2 \beta)}\left(\ln \varepsilon^{-1}\right)^{1 /(1+2 \beta)}$, где $c-$ подходящая постоянная, найдем, что

$$
\begin{aligned}
\Delta_{\varepsilon}^{(\infty)}(\Theta) & \geqslant \sup _{\delta} \delta\left(1-\frac{\mathscr{C}_{\varepsilon}\left(F_{2}\right)-1}{C_{2 \delta}\left(F_{2}\right)+1}\right) \\
& \geqslant c N^{-\beta}\left(1-c \frac{N^{-2 \beta-1}}{\varepsilon^{2} \ln N}\right) \geqslant c \varepsilon^{2 \beta /(1+2 \beta)}\left(\ln \frac{1}{\varepsilon}\right)^{\beta /(1+2 \beta)} .
\end{aligned}
$$

Теорема доказана.

Теорема 6.1 теперь есть очевидное следствие теорем 6.2 и 6.4 .

6.3. В этом пункте мы продолжаем изучение границы $\Delta_{\varepsilon}^{(p)}(\Theta)$ для того случая, когда $\Theta$ определяется границами $L_{2}$-норм смешанных производных. Напомним, что случай $p=2$ был рассмотрен выше, пример 4.1. Мы покажем здесь, что этот результат сохраняется для всех $p<\infty$. Для простоты ограничимся случаем периодических функций $\theta=\theta\left(x_{1}, \ldots, x_{d}\right)$, считая их определенными на кубе $\Gamma=[-\pi, \pi]^{d}$ и периодическими с периодом $2 \pi$. Для функций $\theta \in L_{p}$ определен их ряд Фурье $\theta(x) \sim \sum_{r} \theta_{r} e^{i(x, r)}$, и ниже мы считаем, что для всех функций $\theta \in \Theta$ выполнено условие

$$
\theta_{\tau}=0, \quad \text { если } r_{1} \cdots r_{d}=0, r=\left(r_{1}, \ldots, r_{d}\right) .
$$

Обозначим $S_{p}(\beta, B), \beta=\left(\beta_{1}, \ldots, \beta_{d}\right)$, совокупность всех функций $f \in L_{p}(\Gamma)$, удовлетворяющих условию (6.17) и таких, что

$$
\left\|\frac{\partial^{\beta_{1}+\cdots+\beta_{d}}}{\partial x_{1}^{\beta_{1}} \cdots \partial x_{d}^{\beta_{d}}} f\right\|_{p} \leqslant B .
$$

Теорема 6.5. ПІусть $\Theta \subseteq S_{p}(\beta, B), p<\infty$. Тогда

$$
\Delta_{p}^{(\varepsilon)}(\Theta) \leqslant c \varepsilon^{2 \beta /(2 \beta+1)}\left(\ln \frac{1}{\epsilon}\right)^{\beta(l-1) /(2 \beta+1)},
$$

где $\beta=\min \left(\beta_{1}, \ldots, \beta_{d}\right)$, a $l \geqslant 1$ есть количество $\beta_{j}$, совпадающих $с \beta$. Константа с зависит от $p, \beta, B$.

Д о к а з а т е л ь с т в о следует принщипиальной схеме п. 6.2 и в части, касающейся использования теории приближений, опирается на следуюший результат Б. С. Митягина [7]:

Пусть

$$
f(x) \sim \sum_{r} f_{r} e^{i(r, x)} \in S_{p}(1, \beta) .
$$


Пусть

$$
T_{R} f=\sum_{r} \varphi\left(\frac{\left|r_{1}\right|^{\beta_{1}} \cdots\left|r_{d}\right|^{\beta_{d}}}{R}\right) f_{r} e^{i(r, x)},
$$

где $\varphi(\lambda)$ - финитная бесконечно дифференцируемая функция такая, что $0 \leqslant \varphi \leqslant 1$ и

$$
\varphi(\lambda) \equiv 1 \quad \text { в }|\lambda| \leqslant 1, \quad \varphi(\lambda) \equiv 0 \quad \text { в }|\lambda| \geqslant 2 .
$$

Тогда

$$
\left|f-T_{R} f\right|_{p} \leqslant c_{p}|f|_{p} R^{-1}, \quad p<\infty .
$$

Обозначим $\widetilde{T}_{R}$ оператор, определяемый опять равенством (6.16), где функция $\tilde{\varphi}(\lambda)$ удовлетворяет (6.19) за одним исключением, именно, $\widetilde{\varphi}(0)=0$. Очевидно, что для $f$, удовлетворяющих условию (6.17), $T_{R} f=\tilde{T}_{R} f$.

Оператор $\widetilde{T}_{R}$ - это интегральный оператор с ядром $V_{R}(x-y)$, где $V_{R}(x)$ есть тригонометрический полином

$$
(2 \pi)^{-d} \sum \widetilde{\varphi}\left(\frac{\left|r_{1}\right|^{\beta_{1}} \cdots\left|r_{d}\right|^{\beta_{d}}}{R}\right) e^{i(r, x)}
$$

Оценим $\theta(x)$ посредством

$$
\theta_{R}(x)=\frac{1}{T} \int_{0}^{T} \widetilde{T}_{R}\left(\dot{u}_{\varepsilon}-L u_{\varepsilon}\right) d t
$$

Как и выше (см. п. 6.3 ) и в силу равенства $T_{R} \theta=\widetilde{T}_{R} \theta$

$$
\left|\theta-\theta_{R}\right|_{p} \leqslant\left|\theta-T_{R} \theta\right|_{p}+\varepsilon\left|\frac{1}{T} \int_{0}^{T}\left\langle V_{R}(x-\cdot), d w(t)\right\rangle\right|_{p} .
$$

Интеграл $(\varepsilon / T) \int_{0}^{T}\left\langle V_{R}, d w\right\rangle-$ это случайный тригонометрический полиHOM

$$
t_{r}(x)=\frac{\varepsilon}{\sqrt{T}} \sum \widetilde{\varphi}\left(\frac{\left|r_{1}^{\beta_{1}}\right| \cdots\left|r_{d}^{\beta_{d}}\right|}{R}\right) e^{i(r, x)} \xi_{r},
$$

где $\xi_{r}$ - независимые стандартные гауссовские величины. Поэтому

$$
\begin{array}{r}
\mathbf{E}\left|t_{r}\right|_{p}^{p} \leqslant C^{p} p^{p / 2}\left(\operatorname { c a r d } \left\{r=\left(r_{1}, \ldots, r_{d}\right):\left|r_{j}\right| \geqslant 1,\right.\right. \\
\left.\left.\left|r_{1}^{\beta_{1}}\right| \cdots\left|r_{d}^{\beta_{d}}\right| \leqslant 2 R\right\}\right)^{p / 2}\left(\frac{\varepsilon}{\sqrt{T}}\right)^{p} .
\end{array}
$$

При больших $R$

$$
\operatorname{card}\left\{r:\left|r_{j}\right| \geqslant 1,\left|r_{1}^{\beta_{1}}\right| \cdots\left|r_{d}^{\beta_{d}}\right| \leqslant 2 R\right\} \sim c R^{1 / \beta}(\ln R)^{l-1} .
$$

Следовательно,

$$
\mathbf{E}\left|t_{r}\right|_{p} \leqslant C \sqrt{p} \frac{\varepsilon}{\sqrt{T}} R^{1 /(2 \beta)}(\ln R)^{(l-1) / 2}
$$


Выбирая здесь $R=(\varepsilon / \sqrt[3]{T})^{-2 \beta /(2 \beta+1)}(\ln (\sqrt{T} / \varepsilon))^{-(l-i) 2 \beta / 2(2 \beta+1)}$, найдем из $(6.20)$ и $(6.21)$, что

$$
\Delta_{\varepsilon}^{(p)}(\Theta) \leqslant c \sqrt{p}\left(\frac{\varepsilon}{\sqrt{T}}\right)^{2 \beta /(2 \beta+1)}\left(\ln \frac{\sqrt{T}}{\varepsilon}\right)^{(l-1) \beta /(2 \beta+1)} .
$$

Перейдем к оценкам снизу. Они достигаются с помошью следующей теоремы.

Теорема 6.6. Пусть для какой-нибудь пары $(\beta, B) \Theta \supseteq S_{p}(B, \beta)$, $2 \leqslant p<\infty$. Toгda

$$
\Delta_{\varepsilon}^{(p)}(\Theta) \geqslant c_{p} \varepsilon^{2 \beta /(2 \beta+1)}\left(\ln \frac{1}{\varepsilon}\right)^{(l-1) \beta /(2 \beta+1)}, \quad c_{p}>0 .
$$

Доказательство следует идеям п. 6.2. Будем считать $\beta_{1}=\cdots=\beta_{l}=\beta<\beta_{l+1} \leqslant \cdots \leqslant \beta_{d}$. Рассмотрим функции

$$
\varphi_{m}(x)=\prod_{1}^{l} \cos m_{j} x_{j} \prod_{l+1}^{d} \cos x_{j}
$$

где вектор $m=\left(m_{1}, \ldots, m_{d}\right)$ таков, что $m_{j} \geqslant 1$, а $m_{1} \cdots m_{d} \leqslant N$. Выбор большого числа $N$ мы уточним позднее. Общее число таких функций $M \sim c N(\ln N)^{l-1}$. Рассмотрим теперь функции

$$
f_{\varepsilon}(x)=\frac{N^{-\beta}}{\sqrt{M}} \sum_{m} \varepsilon_{m} \varphi_{m}(x)
$$

где $\varepsilon_{m}= \pm 1$. Обшее число функций $f_{\varepsilon}$ есть $2^{M}$. Покажем, что бо́льшая часть их лежит в $S_{p}(\cdot, \beta)$. С этой целью будем на некоторое время рассматривать коэффициенты $\left\{\varepsilon_{m}\right\}$ как одинаково распределенные независимые случайные величины, принимающие с равными вероятностями значения \pm 1 .

По неравенству Хинчина (см., например, [24])

$$
\mathrm{E}\left|\sum_{m} a_{m} \xi_{m}\right|^{p} \leqslant K^{p} p^{p / 2}\left(\sum a_{m}^{2}\right)^{p / 2},
$$

где $K$ - постоянная. В частности,

$$
\mathbf{E}\left|\frac{\partial^{\beta_{1}}}{\partial x_{1}^{\beta_{1}}} \cdots \frac{\partial^{\beta_{d}}}{\partial x_{d}^{\beta_{d}}} f_{\varepsilon}(x)\right|_{p}^{p} \leqslant K^{p} p^{p / 2}
$$

и, значит,

$$
\mathbf{E}\left|\frac{\partial^{\beta_{1}+\cdots+\beta_{d}}}{\partial x_{1}^{\beta_{1}} \cdots \partial x_{d}^{\beta_{d}}} f_{\varepsilon}\right|_{p} \leqslant K \sqrt{p}
$$

По неравенству Чебышева

$$
\mathbf{P}\left\{\left|\frac{\partial^{\beta_{1}+\cdots+\beta_{d}}}{\partial x_{1}^{\beta_{1}} \cdots \partial x_{d}^{\beta_{d}}} f_{\varepsilon}\right|_{p}>B\right\} \leqslant \frac{K \sqrt{p}}{B} .
$$


Следовательно, умножая, если нужно, $f_{\varepsilon}$ на множитель $\lambda=\lambda(B)$, можно добиться того, что $\mathbf{P}\left\{\lambda f_{\varepsilon} \in S_{p}(B, \beta)\right\} \geqslant \frac{1}{2}$. Последнее неравенство означает, что, по крайней мере, $2^{m-1}$ из $2^{m}$ функций $\lambda f_{\varepsilon}$ лежат в $S_{p}(B, \beta)$. Обозначим этот последний набор функций $f_{\varepsilon}$ через $F$ и будем для простоты считать $\lambda=1$. Будем обозначать $F$ также и множество соответствующих векторов $\varepsilon$. Если $\varepsilon, \varepsilon^{\prime} \in F$, то для $p \geqslant 2$

$$
\begin{aligned}
\left|f_{\varepsilon}-f_{\varepsilon^{\prime}}\right|_{p} & \geqslant(2 \pi)^{1 / p-1 / 2}\left|f_{\varepsilon}-f_{\varepsilon^{\prime}}\right|_{2}=(2 \pi)^{1 / p} \frac{N^{-\beta}}{\sqrt{M}}\left(\sum_{m}\left|\varepsilon_{m}-\varepsilon_{m}^{\prime}\right|^{2}\right)^{1 / 2} \\
& =(2 \pi)^{1 / p} \frac{N^{-\beta}}{\sqrt{M}}\left|\varepsilon-\varepsilon^{\prime}\right| .
\end{aligned}
$$

Пусть $\varepsilon^{0}-$ фиксированный вектор из $F$. Число точек в шаре $U_{\gamma}=$ $\left\{\varepsilon:\left|\varepsilon^{0}-\varepsilon\right| \leqslant \gamma \sqrt{M}\right\}: \operatorname{card} U_{\gamma}=\sum_{k \leqslant \gamma M / 2} C_{M}^{k}$. Обозначим $\mu_{M}$ число успехов в серии из $M$ испытаний Бернулли с вероятностью успеха $\frac{1}{2}$. Тогда

$$
\operatorname{card} U_{\gamma}=2^{M} \sum_{k \leqslant \gamma M / 2} 2^{-M} C_{M}^{k}=2^{M} \mathbf{P}\left\{\mu_{M}<\frac{\gamma M}{2}\right\} .
$$

Из элементарных теорем о больших уклонениях (неравенства Бернштейна или Хеффдинга) следует, что найдется число $c<1$ такое, что card $U_{\gamma} \leqslant 2^{c M}$. Поэтому в $F$ найдется его подмножество $F_{1} \subseteq F$, содержащее не менее $\frac{1}{2} 2^{M(1-c)}$ точек, такое, что для всех $f_{\varepsilon}, f_{\varepsilon^{\prime}} \in F_{1}$

$$
\left|f_{\varepsilon}-f_{\varepsilon^{\prime}}\right|_{p} \geqslant(2 \pi)^{1 / p} \gamma N^{-\beta} \text {. }
$$

Пусть теперь $\delta=\frac{1}{2}(2 \pi)^{1 / p} \gamma N^{-\beta}$. Тогда

$$
\Delta_{p}^{(\varepsilon)}(\Theta) \geqslant \delta\left(1-\frac{\mathscr{C}_{\varepsilon}\left(F_{1}\right)+1}{C_{2 \delta}\left(F_{1}\right)-1}\right) .
$$

Мы видели, что $C_{2 \delta}\left(F_{1}\right) \geqslant M(1-c)-1$. В силу (4.3) $\mathscr{C}_{\varepsilon}\left(F_{1}\right) \geqslant$ $c \pi^{d} /\left(2 N^{2 \beta} \varepsilon^{2}\right)$. Поэтому выбирая $N \asymp \varepsilon^{-2 /(2 \beta+1)}(\ln (1 / \varepsilon))^{-(l-1) /(2 \beta+1)}$, придем к неравенству теоремы.

Соединив вместе неравенства двух предыдущих теорем, мы придем к теореме 6.7.

Теорема 6.7. Пусть $\Theta=S_{p}(B, \beta)$ для каких-нибудь $B, \beta$. Тогда

$$
\Delta_{\varepsilon}^{(p)}(\Theta) \asymp \varepsilon^{2 \beta /(2 \beta+1)}\left(\ln \frac{1}{\varepsilon}\right)^{\beta(l-1) /(2 \beta+1)}, \quad 2 \leqslant p<\infty .
$$

Заметим, что случай $p=\infty$ остался неразобранным. Можно ожидать, что $\Delta_{\varepsilon}^{(\infty)}(\Theta) \asymp \varepsilon^{2 \beta /(2 \beta+1)}(\ln (1 / \varepsilon))^{l \beta /(2 \beta+1)}$.

\section{СПИСОК ЛИТЕРАТУРЫ}

1. Колмогоров A.H., Тихомиров В.M. $\varepsilon$-энтропия и $\varepsilon$-емкость множеств в функциональных пространствах. - Успехи матем. наук, 1959, т. 14, в. 2, с. 3-86. 
2. Ибрагимов И. А., Хасьминский Р. 3. Асимптотическая теория оценивания. М.: Hayкa, 1979, $528 \mathrm{c}$.

3. Ibragimov I., Khasminskii $R$. Some estimation problems in infinite dimensional Gaussian white noise. - In: Festschrift for L. Le Cam: Research papers in probability and statistics. Ed. by Pollard D. et al. New York: Springer-Verlag, 1997, p. 259-274.

4. Гельфаяд И.М., Яалом А.М. О вычислении количества информации о случайной функции, содержашейся в другой такой функции. - Успехи матем. наук, 1957 , т. 12 , в. 1, c. $3-52$.

5. Cover T., Thomas J. Information Theory. New York: Wiley, 1991.

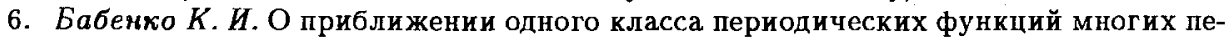
ременных тригонометрическими многочленами. - Докл. АН СССР, 1960, т. 132 , № 5 , с. $982-985$.

7. Митягия $5 . C$. Приближение функций в пространствах $L^{p}$ и $C$ и на торе. Матем. сб., 1962, т. 58, № 4, с. 397-414.

8. Temljakov V.N. Approximation of Periodic Functions. New York: Nova Sci. Publ., 1993.

9. Тихомиров В. М. Поперечники множеств в функциональных пространствах и теория наилучших приближений. - Успехи матем. наук, 1960, т. 15, в. 3, с. 81120 .

10. Тихомиров В. М. Некоторые вопросы теории приближений. М.: Изд-во МГУ, 1976.

11. Ченчов H. Н. Оценка неизвестной плотности распределения по наблюдениям. Докл. АН СССР, 1962, т. 147, № 1, с. 45-48.

12. Agmon S. Lectures on Elliptic Boundary Value Problems. Princeton, NJ: Van Nostrand, 1965, $291 \mathrm{p}$.

13. Jerome J. W. Asymptotic estimates of the $n$-widths in Hilbert space. - Proc. Amer. Math. Soc., 1972, v. 33, № 2, p. 367-372.

14. Пинскер $M$. С. Оптимальная фильтрация квадратично-интегрируемых сигналов на фоне гауссовского шума. - Проблемы передачи информации, 1980, т. 16, № 2 , c. 52-68.

15. Belitser E., Levit $B$. On minimax filtering over ellipsoids. Preprint 863. Utrecht: Dept. Math. Univ. Utrecht, 1994.

16. Ченцов Н. Н. Об оценке неизвестного среднего многомерного нормального распределения. - Теория вероятн. и ее примен., 1967, т. 12, № 4, с. 619-634.

17. Ибрагимов И.А., Хасьминский $Р$. З. Об оценке бесконечномерного параметра в гауссовском белом шуме. - Докл. АН СССР, 1977, т. 236, №6, с. 1053-1055.

18. Никольский C. М. Приближение функцй многих переменных и теоремы вложения. М.: Наука, 1977.

19. Тимая $A . \Phi$. Теория приближения функций действительного переменного. М.: Физматгиз, 1960.

20. Стейн $E$. Сингулярные интегралы и дифференциальные свойства функций. М.: Мир, 1973.

21. Бесов О.В., Ильия В.П. Естественное расширение класса областей в теоремах вложения. - Матем. сб., 1968, т. 75, № 4, с. 483-495.

22. Ибрагимов И.А., Хасьминский Р. З. Об оценке плотности распределения. Зап. научн. семинаров ЛОМИ, 1980, т. 98, с. 61-85.

23. Галлагер $P$. Теория информации и надежная связь. М.: Советское радио, 1974.

24. IIIиряев A. Н. Вероятность. М.: Наука, 1980, 575 с.

25. Ибрагимов И. А., Хасьминский $Р$. З. Задачи оценивания коэффициентов стохастических дифференциальных уравнений в частных производных. I. - Теория вероятн. и ее примен., 1998, т. 43, в. 3, с. 417-438. 